\title{
The ABC of p-Cells
}

\author{
Lars Thorge Jensen
}

\begin{abstract}
Parallel to the very rich theory of Kazhdan-Lusztig cells in characteristic 0, we try to build a similar theory in positive characteristic. We study cells with respect to the $p$-canonical basis of the Hecke algebra of a crystallographic Coxeter system (see [JW17]). Our main technical tool are the star-operations introduced by Kazhdan-Lusztig in [KL79] which have interesting numerical consequences for the $p$-canonical basis. As an application, we explicitely describe $p$-cells in finite type $A$ (i.e. for symmetric groups) using the Robinson-Schensted correspondence. Moreover, we show that Kazhdan-Lusztig cells in finite types $B$ and $C$ decompose into $p$-cells for $p>2$.
\end{abstract}

\section{Contents}

1 Introduction 1

1.1 Structure of the Paper . . . . . . . . . . . . . . . . . . . . . 3

1.2 Acknowledgements . . . . . . . . . . . . . . . . . . . . . 3

2 Background 4

2.1 Coxeter Systems and Generalized Cartan Matrices . . . . . . . . . . . . . . . . . 4

2.2 The Hecke Algebra . . . . . . . . . . . . . . . . . . . . . . . . . . . . . 5

2.3 The Diagrammatic Category of Soergel Bimodules . . . . . . . . . . . . . . . . 5

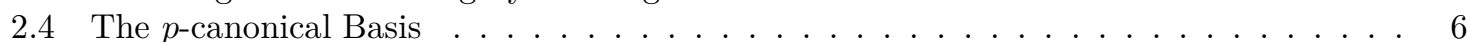

3 General $p$-Cell Theory $\quad 7$

3.1 First Results . . . . . . . . . . . . . . . . . . . . . . . . 7

3.2 Proof of Theorem 3.9 and Corollary $3.10 \ldots \ldots \ldots \ldots \ldots$

3.3 Decomposition Criterion for Kazhdan-Lusztig Cells . . . . . . . . . . . . . . . . . 11

3.4 (Counter-)Examples . . . . . . . . . . . . . . . . . . . . . . . . . 14

4 Left and Right Star Operations 18

4.1 Definition and Numerical Consequences _ . . . . . . . . . . . . . . . . . . . 18

4.2 Consequences for $p$-Cells . . . . . . . . . . . . . . . . . . . . . 24

4.3 Vogan's Generalized $\tau$-Invariant . . . . . . . . . . . . . . . . . . . . . . . . . . . . . 29

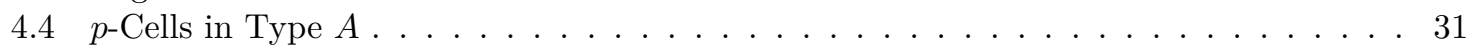

\section{Introduction}

The Hecke algebra of a crystallographic Coxeter system admits several geometric or algebraic categorifications (see [KL79; EW16]). In these cases the resulting canonical basis gives rise to the famous Kazhdan-Lusztig basis (see [KL80; EW14]) in the characteristic 0 setting. The original motivation for the Kazhdan-Lusztig basis was to explicitly construct representations of the Hecke algebra (see 
[Lus18, comments on [37]]). This naturally led Kazhdan and Lusztig to study cells in the Hecke algebra with respect to the Kazhdan-Lusztig basis and the corresponding cell modules.

In [JW17] the p-canonical basis for the Hecke algebra of a crystallographic Coxeter system was introduced. It should be thought of as a positive characteristic analogue of the Kazhdan-Lusztig basis. The $p$-canonical basis shares strong positivity properties with the Kazhdan-Lusztig basis (similar to the ones described by the Kazhdan-Lusztig positivity conjectures), but it loses many of its combinatorial properties. For this reason, it is much harder to compute the $p$-canonical basis which is only known in small examples.

Even without explicit knowledge of the $p$-canonical basis, one may obtain a first approximate description of the multiplicative structure by studying the left, right or two-sided cell preorder with respect to the $p$-canonical basis. Replacing the Kazhdan-Lusztig basis by the $p$-canonical basis in the definition of the left (resp. right or two-sided) cells leads to the notion of left (resp. right or two-sided) $p$-cells. In this paper, we introduce $p$-cell theory which we hope will eventually turn out to be as influential as Kazhdan-Lusztig cell theory. It is current work of progress to describe $p$-cells in affine Weyl groups of small rank. For affine Weyl groups $p$-cells are closely related to tensor ideals of tilting modules (see for example [And04; AHR17; AHR18]).

The first properties of $p$-cells that we prove in Section 3 are the following: Left and right $p$ cells are related by taking inverses (see Lemma 3.6), just like for Kazhdan-Lusztig cells. The set of elements with a fixed left descent set decomposes into right $p$-cells (see Lemma 3.4). We also study which automorphisms of the Hecke algebra are well-behaved with respect to the $p$-canonical basis (see Proposition 3.7). The most important result of this section is a certain compatibility of $p$-cells with parabolic subgroups: We show that any right $p$-cell preorder relation in a finite, standard parabolic subgroup $W_{I}$ induces right $p$-cell preorder relations in each right $W_{I}$-coset (see Theorem 3.9).

In [KL79, Theorem 1.4], Kazhdan and Lusztig show that in type $A$ the cell modules give the irreducible modules of the Hecke algebra $\mathcal{H}$ for generic parameter $v$. In their proof, they introduce the Kazhdan-Lusztig star-operations, generalizing the (dual) Knuth operations for symmetric groups to pairs of simple reflections $s$ and $t$ in a Coxeter group with st of order 3 . The study of the consequences of the star-operations for the structure coefficients leads to an explicit description of the Kazhdan-Lusztig cells in symmetric groups (see [KL79, §5]). The Kazhdan-Lusztig cells in type $A$ can be characterized via the Robinson-Schensted correspondence (see [Ari00]), which gives a bijection $w \mapsto(P(w), Q(w))$ between the symmetric group $S_{n}$ and pairs of standard tableaux of the same shape with $n$ boxes: The Kazhdan-Lusztig right cell of a given element $w \in S_{n}$ is given by the set of elements in $S_{n}$ that have the same $P$-symbol as $w$ under the Robinson-Schensted correspondence.

In an attempt to explicitly describe $p$-cells for symmetric groups, we studied the consequences of the Kazhdan-Lusztig star-operations for the $p$-canonical basis. We deduce many interesting relations for the structure coefficients of the $p$-canonical basis and for the base change coefficients between the $p$-canonical and the Kazhdan-Lusztig basis. The reader is invited to compare these with the results in $[\mathrm{KL} 79, \S 4]$ and [Lus85, §10.4]. If a $p$-canonical basis element differs from the corresponding Kazhdan-Lusztig basis element, then these identities often allow to deduce the non-triviality of other $p$-canonical basis elements (see [LW18, Remark 5.2. (9)] for an example). Another consequence is that $p$-cells coincide with Kazhdan-Lusztig cells in symmetric groups for all primes $p$ (see Theorem 4.33) and in particular that they are independent of $p$. This is particularly interesting since the $p$-canonical basis does differ from the Kazhdan-Lusztig basis for many primes $p$ (see [Wil17b]).

Thus, in type $A$ the $p$-canonical basis of each cell module gives after extending scalars to $\mathbb{C}$ and specializing $v$ to 1 a basis of the corresponding complex irreducible representation of the symmetric group. Letting $p$ vary, we obtain a very interesting family of bases that merits further study. The relation between Specht modules, the Kazhdan-Lusztig cell representations, and their corresponding natural bases was further studied for the Hecke algebra as well as for the group algebra of symmetric groups in [Mat94; GM88; MP05].

Supported by the results in finite type $A$, one may hope that Kazhdan-Lusztig cells always 
decompose into $p$-cells. Unfortunately, this is not the case as we show in Section 3.4.3. However, we believe that the corresponding statement may still be true for $p$ good for the corresponding algebraic group. In Section 3.3, we develop a simple criterion when Kazhdan-Lusztig right cells decompose into right $p$-cells, which reduces the question to minimal elements with respect to the weak right Bruhat order in each cell.

In a series of papers [Gar90; Gar92; Gar93; Gar], Garfinkle generalizes the Robinson-Schensted correspondence to types $B, C$ and $D$. She develops combinatorial algorithms to associate to a Weyl group element $w$ a pair $\left(T_{L}(w), T_{R}(w)\right)$ of standard domino tableaux of the same shape from which $w$ can be reconstructed. (Note that the definition of a standard domino tableau depends on the type.) The main difference to the situation in type $A$ is that the partition of the Weyl group into sets with the same left domino tableau is finer than the partition into Kazhdan-Lusztig left cells (see [McG96, §3]). For this reason, Garfinkle groups the set of dominoes in a tableau into cycles and classifies them as "open" or "closed". For each open cycle, she defines an involutive algorithm called "moving a tableau through an open cycle" that changes only the positions of the dominoes in the open cycle. Based on this, she defines an equivalence relation on the set of standard domino tableaux by declaring two to be equivalent if one can be obtained from the other by moving through a particular subset of open cycles. (In type $B / C$ only the open cycle containing the domino with label 1 is excluded.) One of the main results is that two elements in a Weyl group of type $B / C$ lie in the same Kazhdan-Lusztig left cell if and only if their corresponding left standard domino tableaux are equivalent (see [Gar93, Corollary 3.5.6. and Theorem 3.5.9.]). Unpublished work of Garfinkle (see [Gar]), Pietraho and McGovern extends this result to type $D$. Moreover, Garfinkle shows that a generalization of Vogan's $\tau$-invariant gives a complete invariant for Kazhdan-Lusztig left cells. Based on our results on the Kazhdan-Lusztig star operations, we can show that the equivalence classes with respect to this generalized $\tau$-invariant give a refinement of the left $p$-cells under small assumptions on $p$. This implies, that Kazhdan-Lusztig left cells decompose into left $p$-cells in finite types $B$ and $C$ for $p>2$.

\subsection{Structure of the Paper}

Section 2 We introduce notation and recall important results about the Hecke algebra and the $p$-canonical basis.

Section 3 We define $p$-cells and prove some of their elementary properties. The most important results are the compatibility of $p$-cells with parabolic subgroups and a criterion for KazhdanLusztig cells to decompose into $p$-cells. We also give interesting examples of $p$-cells and state a conjecture resulting from extensive computer calculations.

Section 4 We introduce the Kazhdan-Lusztig star operations. Then we study in detail consequences for base change and structure coefficients of the $p$-canonical basis and for $p$-cells. After introducing Vogan's generalized $\tau$-invariant, we show that left $p$-cells give a refinement of the $\tau$-equivalence classes under small assumptions on $p$. In the end, we show that $p$-cells in finite type $A$ are given by the Robinson-Schensted correspondence.

\subsection{Acknowledgements}

Since this paper is part of the author's PhD-thesis, I would like to thank my supervisor Geordie Williamson - his constant support, his inspiring mathematical vision and his enthusiasm were crucial for the success of my PhD project. Moreover, I am grateful to the Max-Planck Institute for Mathematics for the perfect working conditions and the financial support for my research visit in Sydney. The project was started at the School of Mathematics and Statistics at the University of Sydney and finished at the Max-Planck Institute for Mathematics in Bonn. I would also like to thank Simon 
Riche for detailed comments and Monty McGovern for providing a preliminary version of [Gar] and of his joint work in progress with Thomas Pietraho.

\section{Background}

\subsection{Coxeter Systems and Generalized Cartan Matrices}

Let $S$ be a finite set and $\left(m_{s, t}\right)_{s, t \in S}$ be a matrix with entries in $\mathbb{N} \cup\{\infty\}$ such that $m_{s, s}=1$ and $m_{s, t}=m_{t, s} \geqslant 2$ for all $s \neq t \in S$. Denote by $W$ the group generated by $S$ subject to the relations $(s t)^{m_{s, t}}=1$ for $s, t \in S$ with $m_{s, t}<\infty$. We say that $(W, S)$ is a Coxeter system and $W$ is a Coxeter group. The Coxeter group $W$ comes equipped with a length function $l: W \rightarrow \mathbb{N}$ and the Bruhat order $\leqslant$ (see [Hum90] for more details). A Coxeter system $(W, S)$ is called crystallographic if $m_{s, t} \in\{2,3,4,6, \infty\}$ for all $s \neq t \in S$. We denote the identity of $W$ by $e$. For $w \in W$ we define its left descent set via

$$
\mathcal{L}(w):=\{s \in S \mid l(s w)<l(w)\} .
$$

The right descent set of $w$ is given by $\mathcal{R}(w):=\mathcal{L}\left(w^{-1}\right)$.

Define an expression to be a finite sequence of elements in $S$. We denote by

$$
\operatorname{Ex}(S):=\{\varnothing\} \cup \bigcup_{i \in \mathbb{N} \backslash\{0\}} \underbrace{S \times \cdots \times S}_{i \text {-times }}
$$

the set of all expressions in $S$. For an expression $\underline{w}=\left(s_{1}, s_{2}, \ldots, s_{n}\right)$ denote its length by $l(\underline{w})=n$. For two expressions $\underline{x}$ and $y$ in $S$, we write $\underline{x} \underline{y}$ for their concatenation. The multiplication gives a canonical map $\operatorname{Ex}(S) \rightarrow W, \underline{w} \mapsto \underline{w}$. An expression $\underline{w}$ in $S$ is called reduced if $l(\underline{w})=l\left(\underline{w}_{\bullet}\right)$. For an expression $\underline{w}=\left(s_{1}, s_{2}, \ldots, s_{n}\right)$ in $S$ a subexpression of $\underline{w}$ is a sequence $\underline{w} \underline{e}=\left(s_{1}^{e_{1}}, s_{2}^{e_{2}}, \ldots, s_{n}^{e_{n}}\right)$ where $e_{i} \in\{0,1\}$ for all $i$. The sequence $\underline{e}=\left(e_{1}, e_{2}, \ldots, e_{k}\right)$ is called the associated $01-$ sequence. We usually decorate $\underline{e}$ as follows: For $1 \leqslant k \leqslant n$ denote by $\underline{w}_{\leqslant k}:=\left(s_{1}, s_{2}, \ldots, s_{k}\right)$ the first $k$ terms and set $w_{k}:=\left(\underline{w}^{\underline{e} \leqslant k}\right)$. Assign to $e_{i}$ a decoration $d_{i} \in\{U, D\}$ where $U$ stands for $U p$ and $D$ for Down as follows:

$$
d_{1}:=U \text { and } d_{i}:=\left\{\begin{array}{ll}
U & \text { if } w_{i-1} s_{i}>w_{i-1}, \\
D & \text { if } w_{i-1} s_{i}<w_{i-1}
\end{array} \text { for } 2 \leqslant i \leqslant n .\right.
$$

We often write the decorated sequence as $\left(d_{1} e_{1}, d_{2} e_{2}, \ldots, d_{n} e_{n}\right)$. The sequence of elements $e, w_{1}, w_{2}$, $\ldots, w_{n}$ is called the Bruhat stroll associated to $\underline{w} \underline{\underline{e}}$. The defect of $\underline{e}$ is defined to be

$$
\operatorname{df}(e):=\left|\left\{i \mid d_{i} e_{i}=U 0\right\}\right|-\left|\left\{i \mid d_{i} e_{i}=D 0\right\}\right| .
$$

Example 2.1. To illustrate the definitions, consider for example the case $S=\{s, t\}$ with $m_{s, t}=m_{t, s}=$ 3 (i.e. type $\left.A_{2}\right)$. The reduced expression $(s, t, s)$ admits two decorated 01-sequences expressing $s$ :

$$
\begin{array}{ll}
(U 1, U 0, D 0) & \text { of defect } 0 \\
(U 0, U 0, U 1) & \text { of defect } 2
\end{array}
$$

From now on, fix a generalized Cartan matrix $A=\left(a_{i, j}\right)_{i, j \in J}$ (see [Tit89, §1.1]). Let $\left(J, X,\left\{\alpha_{i}\right.\right.$ : $\left.i \in J\},\left\{\alpha_{i}^{\vee}: i \in J\right\}\right)$ be an associated Kac-Moody root datum (see [Tit89, §1.2] for the definition). Then $X$ is a finitely generated free abelian group, and for $i \in J$ we have elements $\alpha_{i}$ and $\alpha_{i}^{\vee}$ of $X$ and $X^{\vee}=\operatorname{Hom}_{\mathbb{Z}}(X, \mathbb{Z})$ respectively that satisfy $a_{i, j}=\alpha_{i}^{\vee}\left(\alpha_{j}\right)$ for all $i, j \in J$.

To $A$ we associate a crystallographic Coxeter system $(W, S)$ as follows: Choose a set of simple reflections $S$ of cardinality $|J|$ and fix a bijection $S \stackrel{\sim}{\rightarrow} J, s \mapsto i_{s}$. For $s \neq t \in S$ we define $m_{s, t}$ to be $2,3,4,6$, or $\infty$ if $a_{i_{s}, j_{s}} a_{j_{s}, i_{s}}$ is $0,1,2,3$, or $\geqslant 4$ respectively. 
Fix a commutative ring $k$. In both cases, ${ }^{k} V:=X^{\vee} \otimes_{\mathbb{Z}} k$ yields a balanced, potentially non-faithful realization of the Coxeter system over $k$. Set ${ }^{k} V^{*}:=\operatorname{Hom}_{k}\left({ }^{k} V, k\right)$ and note that ${ }^{k} V^{*}$ is isomorphic to $X \otimes_{\mathbb{Z}} k$. A realization obtained in this way is called a Cartan realization (see [AMRW17a, §10.1]). Throughout, we will assume our realization to satisfy:

Assumption 2.2 (Demazure Surjectivity). The maps $\alpha_{s}:{ }^{k} V \rightarrow k$ and $\alpha_{s}^{\vee}:{ }^{k} V^{*} \rightarrow k$ are surjective for all $s \in S$.

This is automatically satisfied if 2 is invertible in $k$ or if the Coxeter system $(W, S)$ is of simply-laced type and of rank $|S| \geqslant 2$.

We denote by $R=S\left({ }^{k} V^{*}\right)$ the symmetric algebra of ${ }^{k} V^{*}$ over $k$ and view it as a graded ring with ${ }^{k} V^{*}$ in degree 2. Given a graded $R$-bimodule $B=\bigoplus_{i \in \mathbb{Z}} B^{i}$, we denote by $B(1)$ the shifted bimodule with $B(1)^{i}=B^{i+1}$.

\subsection{The Hecke Algebra}

The Hecke algebra is the free $\mathbb{Z}\left[v, v^{-1}\right]$-algebra with $\left\{H_{w} \mid w \in W\right\}$ as basis, called the standard basis, and multiplication determined by:

$$
\begin{aligned}
H_{s}^{2} & =\left(v^{-1}-v\right) H_{s}+1 & & \text { for all } s \in S, \\
H_{x} H_{y} & =H_{x y} & & \text { if } l(x)+l(y)=l(x y) .
\end{aligned}
$$

There is a unique $\mathbb{Z}$-linear involution $\overline{(-)}$ on $\mathcal{H}$ satisfying $\bar{v}=v^{-1}$ and $\overline{H_{x}}=H_{x^{-1}}^{-1}$. The Kazhdan-Lusztig basis element $\underline{H}_{x}$ is the unique element in $H_{x}+\sum_{y<x} v \mathbb{Z}[v] H_{y}$ that is invariant under $\overline{(-)}$. This is Soergel's normalization from [Soe97] of a basis introduced originally in [KL79]. For a sequence $\underline{w}=\left(s_{1}, s_{2}, \ldots, s_{n}\right)$ in $S$, we write $\underline{H}_{\underline{w}}$ for the element $\underline{H}_{s_{1}} \underline{H}_{s_{2}} \ldots \underline{H}_{s_{n}}$. After fixing a reduced expression $\underline{w}$ of every element $w \in W$, the set $\left\{\underline{H}_{w} \mid w \in W\right\}$ gives a basis of $\mathcal{H}$, called the Bott-Samelson basis.

Let $\iota$ be the $\mathbb{Z}\left[v, v^{-1}\right]$-linear anti-involution on $\mathcal{H}$ satisfying $\iota\left(H_{s}\right)=H_{s}$ for $s \in S$ and thus $\iota\left(H_{x}\right)=H_{x^{-1}}$.

\subsection{The Diagrammatic Category of Soergel Bimodules}

In this section, we introduce the diagrammatic category of Soergel bimodules. The main reference for this is [EW16] (see also [Eli16] in the dihedral case and [EK10] in type $A$ ).

Let BS be the diagrammatic category of Bott-Samelson bimodules as introduced in [JW17, §2.3]. It is a diagrammatic, strict monoidal category enriched over $\mathbb{Z}$-graded left $R$-modules.

Let $\mathbf{H}$ be the Karoubian envelope of the graded version of the additive closure of BS, in symbols $\mathbf{H}=\mathcal{K}$ ar $(\mathrm{BS})$. We call $\mathbf{H}$ the diagrammatic category of Soergel bimodules. In other words, in the passage from BS to $\mathbf{H}$ we first allow direct sums and grading shifts (restricting to degree preserving homomorphisms) and then the taking of direct summands. The following properties can be found in [EW16, Lemma 6.24, Theorem 6.25 and Corollary 6.26]:

Theorem 2.3 (Properties of $\mathbf{H})$.

Let $k$ be a complete, local, integral domain (e.g. a field or the p-adic integers $\mathbb{Z}_{p}$ ).

(i) $\mathbf{H}$ is a Krull-Schmidt category.

(ii) For all $w \in W$ there exists a unique indecomposable object ${ }^{k} B_{w} \in \mathbf{H}$ which is a direct summand of $\underline{w}$ for any reduced expression $\underline{w}$ of $w$ and which is not isomorphic to a grading shift of any direct summand of any expression $\underline{v}$ for $v<w$. In particular, the object ${ }^{k} B_{w}$ does not depend up to isomorphism on the reduced expression $\underline{w}$ of $w$. 
(iii) The set $\left\{{ }^{k} B_{w} \mid w \in W\right\}$ gives a complete set of representatives of the isomorphism classes of indecomposable objects in $\mathbf{H}$ up to grading shift.

(iv) There exists a unique isomorphism of $\mathbb{Z}\left[v, v^{-1}\right]$-algebras

$$
\operatorname{ch}:[\mathbf{H}] \longrightarrow \mathcal{H}
$$

sending $\left[{ }^{k} B_{s}\right]$ to $\underline{H}_{s}$ for all $s \in S$, where $[\mathbf{H}]$ denotes the split Grothendieck group of $\mathbf{H}$. (We view $[\mathbf{H}]$ as a $\mathbb{Z}\left[v, v^{-1}\right]$-algebra as follows: the monoidal structure on $\mathbf{H}$ induces a unital, associative multiplication and $v$ acts via $v[B]:=[B(1)]$ for an object $B$ of $\mathbf{H}$.)

It should be noted that we do not have a diagrammatic presentation of $\mathbf{H}$ as determining the idempotents in BS is usually extremely difficult.

\subsection{The $p$-canonical Basis}

In this section, we recall the definition of the $p$-canonical basis and its elementary properties (see [JW17]). Instead of a based root datum, we use a generalized Cartan matrix as input, but all the results from [JW17] still hold in this slightly more general setting. Let $k$ be a field of characteristic $p \geqslant 0$. Note that the $p$-canonical basis depends on $p$, but not on the explicit choice of $k$. Since the numerical input for the algorithm to calculate the $p$-canonical basis (as described in [JW17, §3]) reduces to the generalized Cartan matrix $A$, the $p$-canonical basis does not depend on the choice of Kac-Moody root datum associated to $A$.

Definition 2.4. Define ${ }^{p} \underline{H}_{w}=\operatorname{ch}\left(\left[{ }^{k} B_{w}\right]\right)$ for all $w \in W$ where $\operatorname{ch~}:[\mathbf{H}] \stackrel{\cong}{\longrightarrow} \mathcal{H}$ is the isomorphism of $\mathbb{Z}\left[v, v^{-1}\right]$-algebras introduced earlier.

We will frequently use the following elementary properties of the $p$-canonical basis which can be found in [JW17, Proposition 4.2] unless stated otherwise:

Proposition 2.5. For all $x, y \in W$ we have:

(i) $\overline{{ }^{p} \underline{H}_{x}}={ }^{p} \underline{H}_{x}$, i.e. ${ }^{p} \underline{H}_{x}$ is self-dual,

(ii) ${ }^{p} \underline{H}_{x}=H_{x}+\sum_{y<x}{ }^{p} h_{y, x} H_{y}$ with ${ }^{p} h_{y, x} \in \mathbb{Z}_{\geqslant 0}\left[v, v^{-1}\right]$,

(iii) ${ }^{p} \underline{H}_{x}=\underline{H}_{x}+\sum_{y<x}{ }^{p} m_{y, x} \underline{H}_{y}$ with self-dual ${ }^{p} m_{y, x} \in \mathbb{Z}_{\geqslant 0}\left[v, v^{-1}\right]$,

(iv) $\iota\left(\underline{H}_{x}\right)={ }^{p} \underline{H}_{x^{-1}}$ and thus in particular ${ }^{p} m_{y, x}={ }^{p} m_{y^{-1}, x^{-1}}$ as well as ${ }^{p} h_{y, x}={ }^{p} h_{y^{-1}, x^{-1}}$,

(v) ${ }^{p} m_{y, x}=0$ unless $\mathcal{L}(x) \subseteq \mathcal{L}(y)$ and $\mathcal{R}(x) \subseteq \mathcal{R}(y)$,

(vi) ${ }^{p} \underline{H}_{x}{ }^{p} \underline{H}_{y}=\sum_{z \in W}{ }^{p} \mu_{x, y}^{z} \underline{H}_{z}$ with self-dual ${ }^{p} \mu_{x, y}^{z} \in \mathbb{Z}_{\geqslant 0}\left[v, v^{-1}\right]$,

(vii) ${ }^{p} \underline{H}_{x}=\underline{H}_{x}$ for $p=0$ (see [EW14]) and $p \gg 0$ (i.e. there are only finitely many primes for which ${ }^{p} \underline{H}_{x} \neq \underline{H}_{x}$ ).

Denote by $\mu(y, x)$ the coefficient of $v$ in the Kazhdan-Lusztig polynomial ${ }^{0} h_{y, x}$.

The following result (see [JW17, Lemma 4.3]) shows the remnant of the multiplication formula for the Kazhdan-Lusztig basis. Note that ${ }^{p} \underline{H}_{s}=\underline{H}_{s}$ for all $p \geqslant 0$ as the corresponding Schubert variety is smooth.

Lemma 2.6. For $x \in W$ and $s \in \mathcal{L}(x)$ we have:

$$
\underline{H}_{s}^{p} \underline{H}_{x}=\left(v+v^{-1}\right)^{p} \underline{H}_{x} .
$$




\section{General $p$-Cell Theory}

\section{1 $\quad$ First Results}

In this section, we want to give the definition of $p$-cells. This notion is an obvious generalization of a notion introduced by Kazhdan-Lusztig in [KL79].

Definition 3.1. For $h \in \mathcal{H}$ we say that ${ }^{p} \underline{H}_{w}$ appears with non-zero coefficient in $h$ if the coefficient of ${ }^{p} \underline{H}_{w}$ is non-zero when expressing $h$ in the $p$-canonical basis.

Define a preorder $\underset{R}{\stackrel{p}{\leqslant}}($ resp. $\underset{L}{\stackrel{p}{\leqslant}})$ on $W$ as follows: $x \underset{R}{\stackrel{p}{\leqslant}} y(\operatorname{resp} x \underset{L}{\stackrel{p}{\leqslant}} y)$ if and only if $\underline{H}_{x}$ appears with non-zero coefficient in ${ }^{p} \underline{H}_{y} h\left(\right.$ resp. $\left.h^{p} \underline{H}_{y}\right)$ for some $h \in \mathcal{H}$. Define $\underset{L R}{\stackrel{p}{\leqslant}}$ to be the preorder generated by $\underset{R}{\stackrel{p}{\leqslant}}$ and $\underset{L}{\stackrel{p}{\leqslant}}$, in other words we have: $x \underset{L R}{\stackrel{p}{\leqslant}} y$ if and only if ${ }^{p} \underline{H}_{x}$ appears with non-zero coefficient in $h^{p} \underline{H}_{y} h^{\prime}$ for some $h, h^{\prime} \in \mathcal{H}$.

Restricting $h$ and $h^{\prime}$ in the definitions above to any set of generators of $\mathcal{H}$ as a $\mathbb{Z}\left[v, v^{-1}\right]$-algebra, one gets a set of generating relations for the corresponding $p$-cell preorders (see [Wil03, Proposition 4.1.1]). The following definition introduces some notation for the relations generating the $p$-cell preorder obtained from the generating set $\left\{\underline{H}_{s} \mid s \in S\right\}$ which we will use in Section 4.2.

Definition 3.2. Let $x, y \in W$. We write $x \underset{L}{\stackrel{p}{t}} y$ (resp. $x \underset{R}{\stackrel{p}{L}} y)$ if ${ }^{p} \mu_{s, y}^{x}$ (resp. ${ }^{p} \mu_{y, s}^{x}$ ) is non-zero for some $s \in S$. In addition, we write $x \underset{2}{\stackrel{p}{L}} y$ if $x \underset{L}{\stackrel{p}{L}} y$ or $x \underset{R}{\stackrel{p}{R}} y$ holds.

For the sake of completeness, we will state explicitly that these elementary relations generate the $p$-cell-preorder (see [AHR17, Lemma 5.3] for a proof):

Lemma 3.3. For $x, y \in W$ the following holds: $x \underset{R}{\stackrel{p}{\leqslant}} y$ if and only if there exists a chain $x=x_{0} \underset{R}{\stackrel{p}{R}}$ $x_{1} \underset{R}{\stackrel{p}{\leftarrow}} \ldots \stackrel{p}{\stackrel{p}{R}} x_{k}=y$. Similarly for the left (resp. two-sided) p-cell preorder.

In the remainder of the section, we will prove some elementary properties of $p$-cells. In most cases we will focus on right $p$-cells and not state the version for left $p$-cells explicitly.

In [KL79, Proposition 2.4] Kazhdan-Lusztig observed that a Kazhdan-Lusztig right cell preorder relation implies an inclusion of left descent sets. The following result shows that the compatibility between cells and descent sets carries over to the more general setting. More precisely, the set of all elements with a fixed left descent set is a union of right $p$-cells. The result can also be found in [AHR17, Lemma 5.4]:

Lemma 3.4. For $x, y \in W$ with $y \underset{R}{\stackrel{p}{\leqslant}} x$ we have $\mathcal{L}(x) \subseteq \mathcal{L}(y)$. In particular, $x \underset{R}{\stackrel{p}{\sim}}$ y gives $\mathcal{L}(x)=\mathcal{L}(y)$ and for any $I \subseteq S$ the set $\{w \in W \mid \mathcal{L}(w)=I\}$ is a union of right $p$-cells.

Proof. Due to Lemma 3.3 it is enough to consider the case where we multiply ${ }^{p} \underline{H}_{x}$ with ${ }^{p} \underline{H}_{s}$ for $s \notin \mathcal{R}(x)$. We have on the one hand:

$$
{ }^{p} \underline{H}_{x}^{p} \underline{H}_{s}=\sum_{y}{ }^{p} \mu_{x, s}^{y}{ }^{p} \underline{H}_{y}
$$

On the other hand we can write:

$$
{ }^{p} \underline{H}_{x}^{p} \underline{H}_{s}=\left(\sum_{y \leqslant x}{ }^{p} m_{y, x} \underline{H}_{y}\right) \underline{H}_{s}
$$




$$
=\sum_{\substack{y \leqslant x \\ s \in \mathcal{R}(y)}}\left(v+v^{-1}\right)^{p} m_{y, x} \underline{H}_{y}+\sum_{\substack{y \leqslant x \\ s \notin \mathcal{R}(y)}} m_{y, x}\left(\underline{H}_{y s}+\sum_{\substack{z \leqslant y \\ s \in \mathcal{R}(z)}} \mu(z, y) \underline{H}_{z}\right)
$$

Proposition 2.5(v) shows that all $y \in W$ occurring with non-zero ${ }^{p} m_{y, x}$ on the right hand side satisfy $\mathcal{L}(x) \subseteq \mathcal{L}(y)$. [KL79, Proposition 2.4] shows that $z \underset{R}{\leqslant} y$ implies $\mathcal{L}(y) \subseteq \mathcal{L}(z)$. Observe that the set of $y \in W$ with non-zero structure coefficient ${ }^{p} \mu_{x, s}^{y}$ is a subset of the set of all $y \in W$ indexing a summand $\underline{H}_{y}$ with non-zero coefficient on the right hand side (due to Proposition 2.5(iii) and (vi)). Putting all of this together gives the result.

Corollary 3.5. $\{\mathrm{Id}\}$ is a left, right, and 2-sided $p$-cell for all primes $p$.

It is well known for Kazhdan-Lusztig cells that left and right cells are closely related via taking inverses. Using the $\mathbb{Z}\left[v, v^{-1}\right]$-linear anti-involution $\iota$ on $\mathcal{H}$ together with Proposition 2.5(iv) we obtain the corresponding result for $p$-cells which will allow us to pass from left to right $p$-cells:

Lemma 3.6. For all $x, y \in W$ we have:

$$
\begin{aligned}
& x \underset{L}{\stackrel{p}{\leqslant}} y \Longleftrightarrow x^{-1} \underset{R}{\stackrel{p}{\leqslant}} y^{-1}, \\
& x \underset{L R}{\stackrel{p}{\leqslant}} y \Longleftrightarrow x^{-1} \underset{L R}{\stackrel{p}{\leqslant}} y^{-1} .
\end{aligned}
$$

Next, we want to consider the question which automorphisms of our Coxeter system induce automorphisms on $\mathcal{H}$ that are well-behaved with respect to the $p$-canonical basis. Let $\phi:(W, S) \stackrel{\sim}{\longrightarrow}$ $(W, S)$ be an automorphism of Coxeter systems (in particular we have $\phi(S)=S$ ) which leaves the generalized Cartan matrix $A$ invariant when permuting simultaneously the corresponding rows and columns (i.e. $\left\langle\alpha_{t}^{\vee}, \alpha_{s}\right\rangle=\left\langle\alpha_{\phi(t)}^{\vee}, \alpha_{\phi(s)}\right\rangle$ for all $s, t \in S$ ). Then $\phi$ induces a $\mathbb{Z}\left[v, v^{-1}\right]$-linear automorphism of $\mathcal{H}$ via $H_{x} \mapsto H_{\phi(x)}$ for $x \in W$ which we will also denote by $\phi$ by slight abuse of notation. Therefore, $\phi$ maps $\underline{H}_{x}$ to $\underline{H}_{\phi(x)}$ by the defining property of the Kazhdan-Lusztig basis.

Proposition 3.7. In the setting given above we have for all $x, y \in W$ :

(i) $\phi\left(\underline{H}_{x}\right)={ }^{p} \underline{H}_{\phi(x)}$,

(ii) ${ }^{p} m_{y, x}={ }^{p} m_{\phi(y), \phi(x)}$ and ${ }^{p} h_{x, y}={ }^{p} h_{\phi(x), \phi(y)}$,

(iii) ${ }^{p} \mu_{x, y}^{z}={ }^{p} \mu_{\phi(x), \phi(y)}^{\phi(z)}$,

(iv) $x \underset{L}{\stackrel{p}{\leftarrow}} y \Leftrightarrow \phi(x) \underset{L}{\stackrel{p}{\leqslant}} \phi(y)$ and $x \underset{R}{\stackrel{p}{\leftarrow}} y \Leftrightarrow \phi(x) \underset{R}{\stackrel{p}{\leqslant}} \phi(y)$.

Proof. Observe that $\phi$ induces a monoidal, $k$-linear equivalence of BS and thus of $\mathbf{H}$ which on the Hom-spaces merely permutes the colours in the diagrams (given by $S$ ) and the variables of the polynomials in $R$ decorating the regions according to the action of $\phi$. Since the numerical input for the algorithm to calculate the $p$-canonical basis (as described in [JW17, §3]) reduces to $A$ we see immediately that this equivalence sends ${ }^{k} B_{x}$ to ${ }^{k} B_{\phi(x)}$ and thus on the level of Grothendieck groups ${ }^{p} \underline{H}_{x}$ to ${ }^{p} \underline{H}_{\phi(x)}$. This proves (i).

Recall that $\phi$ maps $\underline{H}_{x}$ to $\underline{H}_{\phi(x)}$ and $H_{x}$ to $H_{\phi(x)}$ for all $x \in W$. For this reason, (ii) follows from (i) by rewriting ${ }^{p} \underline{H}_{x}$ in the Kazhdan-Lusztig basis (resp. standard basis), applying $\phi$, using (i) and comparing coefficients in the Kazhdan-Lusztig basis (resp. standard basis). (i) implies (iii) in a similar way and (iv) follows from (iii). 
Suppose that our based root datum is irreducible. In this case, the last proposition can be applied to all automorphisms of the (extended) Dynkin diagram of our root system. In finite type conjugation by the longest element in the finite Weyl group is also covered by the last proposition. Indeed, it follows from [Dav08, Remark 13.1.8] that for irreducible finite Coxeter groups the longest element $w_{0}$ is central except in types $A_{n}$ for $n \geqslant 2, D_{n}$ with $n$ odd, $E_{6}$, and $I_{2}(m)$ for $m$ odd where $I_{2}(m)$ denotes the dihedral group of order $2 m$. In all these cases, conjugation by $w_{0}$ gives the obvious automorphism of the corresponding Coxeter graph. After restricting to crystallographic Coxeter systems, only simply-laced types remain and so any automorphism of the Coxeter graph gives an automorphism of the Dynkin diagram of the same type in the obvious way (as the graphs are isomorphic).

Definition 3.8. Let $I \subseteq S$ be a subset. Call $I$ finitary if the corresponding parabolic subgroup $\langle I\rangle \subseteq W$ is finite. Define $W^{I}$ to be the set of representatives of minimal length of cosets in $W / W_{I}$.

The following result is the main result of this section and generalizes the parabolic compatibility for Kazhdan-Lusztig cells (see [Lus03, Proposition 9.11]) to the setting of $p$-cells:

Theorem 3.9 (Parabolic compatibility of right $p$-cells).

Let $I \subseteq S$ be a finitary subset. Then for $y, z \in W_{I}$ the following holds:

$$
z \underset{R}{\stackrel{p}{\leqslant}} y \text { in } W_{I} \Leftrightarrow \forall x \in W^{I}: x z \underset{R}{\stackrel{p}{\leqslant}} x y \text { in } W
$$

As a corollary to the proof of Theorem 3.9 we get:

Corollary 3.10. In the setting of Theorem 3.9 we have:

$$
{ }^{p} h_{x y, x z}={ }^{p} h_{y, z}
$$

Remark 3.11. Let $H \subseteq W$ be a finite standard parabolic subgroup and $C \subseteq W$ a right $p$-cell. Theorem 3.9 implies immediately that the cell module associated to $C$ when restricted to $H$ decomposes into cell modules for $H$ (see [BV83, Proposition 3.11] and [Roi98, Proposition 1]).

\subsection{Proof of Theorem 3.9 and Corollary 3.10}

First we will prove Theorem 3.9: For all elements $w$ in $W_{I} \cup W^{I}$ choose a reduced expression $\underline{w}$. We have a bijection

$$
\begin{aligned}
W^{I} \times W_{I} & \longrightarrow W \\
(x, y) & \longmapsto x y
\end{aligned}
$$

such that $l(x y)=l(x)+l(y)$ (see [BB05, Proposition 2.4.4]). Therefore, for $x \in W^{I}$ and $y \in W_{I}$ the concatenation of the corresponding reduced expressions $\underline{x}$ and $\underline{y}$ gives a reduced expression $\underline{x} \neg \underline{y}$ of $x y$. Choose $x \in W^{I}$ and $y \in W_{I}$ arbitrarily. In the following we sometimes identify a subexpression with its associated 01-sequence.

Lemma 3.12. For all $z \in W_{I}$ we have a decoration- and defect-preserving bijection:

$$
\begin{aligned}
g:\{\text { subexpr. of } \underline{y} \text { expressing } z\} & \stackrel{\sim}{\longrightarrow}\{\text { subexpr. of } \underline{x} \underline{y} \text { expressing } x z\} \\
\underline{e} & \underbrace{(1, \ldots, 1)}_{l(x) \text { ones }}\urcorner \underline{e}
\end{aligned}
$$

Proof. It is easy to see that $g$ is well-defined and injective. Leaving out any letter of $\underline{x}$ in a subexpression of $\underline{x} y$ leads to a Bruhat stroll ending in a right coset $\widetilde{x} W_{I}$ with $\widetilde{x}<x$ as $x$ is of minimal length in $x \overline{W_{I}}$. Thus, $g$ is surjective. 
For the definition of the Bruhat graph we refer the reader to [Dye91, Definition 1.1]. The induced Bruhat graph on $x W_{I}$ (as a subgraph of the Bruhat graph of $W$ ) is isomorphic to the one of $W_{I}$ (see [Dye91, Theorem 1.4]). This follows for example from the subword property (see [BB05, Theorem $2.2 .2])$. Thus any subexpression $g(\underline{e})$ of $\underline{x} \underline{y}$ expressing $x z$ will have a decoration starting with $l(x)$ symbols $U 1$ (as $\underline{x}$ is a reduced expression) and the remaining expression $\underline{e}$ will be decorated in the same way as $\underline{e}$ would be decorated as a subexpression of $\underline{y}$ expressing $z$. Since the ones in a subexpression do not contribute to the defect, this immediately implies:

$$
\operatorname{df}(g(\underline{e}))=\operatorname{df}(\underline{e})
$$

where on the left (resp. right) hand side the defect is calculated as a subexpression of $\underline{x} \underline{y}$ (resp. $\underline{y})$.

This bijection matches up the combinatorial data used to define the light leaves and thus allows us to compare the corresponding local intersection forms. Consider the local intersection forms $I_{\underline{x}} \underline{y}, x z$ of $\underline{x} \underline{y}$ at $x z$ (resp. $I_{\underline{y}, z}$ of $\underline{y}$ at $z$ ) and the matrices representing them with respect to the light leaves bases (see $[J W 17, \S 3]$ ). For two subexpressions $\underline{e}, \underline{f}$ of $\underline{y}$ expressing $z$ we get in ${ }^{k} \mathbf{H}^{\nless x z} \otimes_{R} k$ :

$$
\left(I_{\underline{x}}, x z\right)_{g(\underline{e}), g(\underline{f})}=\operatorname{Id}_{\mathrm{BS}(\underline{x})}\left(I_{\underline{y}, z}\right)_{\underline{e}, \underline{f}}
$$

This implies that the multiplicity of ${ }^{k} B_{x z}$ and its grading shifts in $\operatorname{BS}(\underline{x} \underline{y})$ which is given by grk $\left(I_{\underline{x}} \underline{y}, x z\right)$ coincides with the multiplicity of ${ }^{k} B_{z}$ and its grading shifts in $\operatorname{BS}(\underline{y})$ which is given by $\operatorname{grk}\left(I_{\underline{y}, z}\right)$.

Choose any total order on $W$ refining the Bruhat order and preserving elements in the same coset in $W / W_{I}$ as blocks of adjacent elements. Note that our choices above have fixed a reduced expression $\underline{w}$ for any element $w \in W$. Denote by $M$ the base change matrix from the Bott-Samelson basis $\left\{\underline{H}_{w} \mid w \in W_{I}\right\}$ to the $p$-canonical basis $\left\{\underline{H}_{w} \mid w \in W_{I}\right\}$ of $\mathcal{H}_{\left(W_{I}, I\right)} . M$ is an upper-triangular, invertible matrix with entries in $\mathbb{Z}\left[v, v^{-1}\right]$ and ones on the diagonal. The above considerations show that the base change matrix from the Bott-Samelson to the $p$-canonical basis of $\mathcal{H}$ looks as follows:

$$
\begin{gathered}
W_{I} \\
\vdots \\
x W_{I} \\
\vdots
\end{gathered}
$$

Note that this form is preserved by taking inverses. This means that when expressing ${ }^{p} \underline{H}_{x y}$ in terms of the Bott-Samelson basis $M^{-1}$ gives all coefficients for terms indexed by $\underline{x z}$ for $z \in W_{I}$. Using this allows us to partly decouple the terms $\underline{H}_{x}$ and $\underline{H}_{y}$. When calculating ${ }^{p} \underline{H}_{x y} \underline{H}_{w}$ for $w \in W_{I}$ we can simply express both terms in the Bott-Samelson basis, perform the calculation where only the structure coefficients for the Bott-Samelson basis of $\mathcal{H}_{\left(W_{I}, I\right)}$ come into play and rewrite it in terms of the $p$-canonical basis. This immediately implies the following result as we have full control over the situation in the top coset $x W_{I}$ :

Corollary 3.13. For all $y, z, w \in W_{I}$ and a minimal coset representative $x$ of $W / W_{I}$ we have:

$$
{ }^{p} \mu_{x y, w}^{x z}={ }^{p} \mu_{y, w}^{z}
$$

The last corollary proves Theorem 3.9 since elementary relations obtained from ${ }^{p} \underline{H}_{w}^{p} \underline{H}_{s}$ for $w \in W_{I}$ and $s \in I$ generate the right $p$-cell preorder in $W_{I}$ (see Lemma 3.3).

Next, we will prove Corollary 3.10:

Recall the following lemma which in the case of a reduced expression $\underline{w}$ describes how to express the Bott-Samelson basis element $\underline{H}_{\underline{w}}$ in terms of the standard basis (see [EW16, Lemma 2.10]): 
Lemma 3.14. For any expression $\underline{w}$ in $S$ we have:

$$
\underline{H}_{\underline{w}}=\sum_{\substack{\text { e subexpression } \\ \text { of } \underline{w}}} v^{\mathrm{df}(\underline{e})} H_{(\underline{w} \underline{e}) \text {. }}
$$

Recall that we have chosen a total order on $W$ in the proof of Theorem 3.9. Denote by $B$ the base change matrix from the Bott-Samelson basis $\left\{\underline{H}_{w} \mid w \in W_{I}\right\}$ to the standard basis $\left\{H_{w} \mid w \in W_{I}\right\}$ of $\mathcal{H}_{\left(W_{I}, I\right)}$. Then $B$ is an upper-triangular, invertible matrix with entries in $\mathbb{Z}\left[v, v^{-1}\right]$ and ones on the diagonal. The defect-preserving bijection from Lemma 3.12 shows that the base change matrix from the Bott-Samelson to the standard basis of $\mathcal{H}$ looks as follows:

$$
\begin{gathered}
W_{I} \\
\vdots \\
x W_{I} \\
\vdots
\end{gathered}
$$

Multiplying the base change matrix from the $p$-canonical to the Bott-Samelson basis with the base change matrix from the Bott-Samelson to the standard basis finishes the proof of Corollary 3.10.

\subsection{Decomposition Criterion for Kazhdan-Lusztig Cells}

In this section, we want to study the interplay between the weak right Bruhat order (see [BB05, Definition 3.1.1] for the definition) and the right $p$-cell preorder. This will allow us to formulate a simple criterion as to when $p$-cells decompose into Kazhdan-Lusztig cells.

In the next few results we will focus on right cells, but a similar version for left cells can easily be formulated. Throughout this section, we assume that $W$ has finitely many right Kazhdan-Lusztig cells. This is known for finite and affine Weyl groups by [Lus87, Theorem 2.2 (a)], but there are examples of crystallographic Coxeter groups with infinitely many right Kazhdan-Lusztig cells (see [Bé89; Bel04]).

For $x \in W$, let $\underline{x}=\left(s_{1}, s_{2}, \ldots, s_{k}\right)$ be a reduced expression. Set $x_{i}:=s_{1} s_{2} \ldots s_{i}$ for all $0 \leqslant i \leqslant k$. Since ${ }^{p} \underline{H}_{0}=\underline{H}_{e}$ there exists a maximal $0 \leqslant m \leqslant k$ such that for all $y \leqslant x_{m}$ with ${ }^{p} m_{y, x_{m}} \neq 0$ we have $y \underset{R}{\stackrel{0}{\leqslant}} x_{m}$. In this setting we have the following result:

Lemma 3.15. All $y \leqslant x$ with ${ }^{p} m_{y, x}$ non-zero satisfy: $y \stackrel{0}{\leqslant} x_{m}$.

Proof. The claim follows as ${ }^{p} \underline{H}_{x_{l}}$ for $l \geqslant m$ is a linear combination of Kazhdan-Lusztig basis elements indexed by elements in $\left\{w \in W \mid w \stackrel{0}{\leqslant} x_{m}\right\}$.

Observe that $x_{m} \underset{R}{\stackrel{0}{*}} x$ always holds. So if $x_{m} \underset{R}{\stackrel{0}{\leqslant}} x$, then $x$ and $x_{m}$ lie in the same Kazhdan-Lusztig right cell.

Corollary 3.16. If $x_{m}$ and $x$ lie in the same Kazhdan-Lusztig right cell, then for $y \leqslant x$ with ${ }^{p} m_{y, x}$ non-zero we have $y \underset{R}{\stackrel{0}{\leqslant}} x$.

Definition 3.17. Let $C \subseteq W$ be an arbitrary subset. $C$ is called right-connected if for every two elements $x, y \in C$, there exists a sequence $x=x_{0}, x_{1}, \ldots, x_{k}=y$ in $C$ such that $x_{i}^{-1} x_{i-1} \in S$ (i.e. 
$x_{i-1}$ and $x_{i}$ differ by a simple reflection on the right) for all $1 \leqslant i \leqslant k$. It follows that $C$ decomposes as a disjoint union of its right-connected components, i.e. the maximal right-connected subsets.

Call an element $x \in C$ right-minimal if $x$ cannot be reached from any other element $y \in C \backslash\{x\}$ via a sequence $y=x_{0}, x_{1}, \ldots, x_{k}=x$ in $C$ as above satisfying in addition $y<x_{1}<x_{2}<\cdots<x_{k}=x$. Observe that an element is right-minimal if and only if it is minimal with respect to the weak right Bruhat order.

Similarly we define left-connected and left-minimal using multiplication by simple reflections on the left, as well as 2-connected and 2-minimal.

The following observation follows immediately from the definition of a right-minimal element, but shows their most important property:

Lemma 3.18. Let $C \subseteq W$ be an arbitrary subset. For all $y \in C$ there exists a right-minimal element $x \in C$ such that $y \underset{R}{\stackrel{0}{\leqslant}} x$ and $y \underset{R}{\stackrel{p}{\leqslant}} x$.

At this point we should mention the following conjecture by Lusztig which he originally formulated for (affine) Weyl groups in [HK83, p. 14]. It still appears to be open in finite type $D_{n}$ and in general affine type:

\section{Conjecture 3.19.}

Every Kazhdan-Lusztig right cell in a Coxeter group is right-connected.

In finite type, the conjecture is known to hold for all dihedral groups, in type $A_{n}$ (see [KL79, §5]), $B_{n}$ (see [Gar93, Theorem 3.5.9]) and in all exceptional types $H_{3}, H_{4}, F_{4}, E_{6}, E_{7}$ and $E_{8}$ (see [GH15, Example 7.3]). In affine type, it has been verified in affine rank 2 (see [Lus85, Theorem 11.3]), for $W$ of type $\widetilde{A}_{n}$ with $n \geqslant 1$ (see [Shi86, Theorem 18.2.1]), for Kazhdan-Lusztig right cells contained in the lowest Kazhdan-Lusztig two-sided cell (see [Shi88, Corollary 1.2]), for Kazhdan-Lusztig right cells contained in the Kazhdan-Lusztig two-sided cell of elements with a unique reduced expression (see [Lus83, Proposition 3.8]) and some other special cases (see for example [Xi89], [Shi02, Theorem 4.8] and [Shi06]).

In the rest of the section we want to apply these notions to compare Kazhdan-Lusztig and $p$-cells by looking at minimal elements. We will focus on right cells even though there are similar results about left (resp. two-sided) cells. Using a similar idea as in Corollary 3.16 we obtain the following result:

Corollary 3.20. Let $C$ be a Kazhdan-Lusztig right cell and $C_{\min }$ the set of right-minimal elements in $C$. Assume for all $x \in C_{\min }$ and $y \leqslant x$ the following:

$$
{ }^{p} m_{y, x} \neq 0 \Rightarrow y \stackrel{0}{\leqslant} x
$$

Then for all $x \in C$ and $y \leqslant x$ with ${ }^{p} m_{y, x} \neq 0$ we have $y \underset{R}{\stackrel{0}{\leqslant}} x$.

Definition 3.21. Let $X$ be a set equipped with a preorder $\leqslant$. A subset $Y \subseteq X$ is called a lower set if for $y \in Y$ and any $x \in X$ with $x \leqslant y$ we have $x \in Y$ as well.

Observe that any lower set in the right $p$-cell preorder can be written as a union of right $p$-cells. For this reason the following result is the starting point of our criterion:

Lemma 3.22. Let $C$ be a Kazhdan-Lusztig right cell that satisfies the assumptions of Corollary 3.20 and $x \in C$. Then $y \underset{R}{\stackrel{p}{\leqslant}} x$ implies $y \underset{R}{\stackrel{0}{\leqslant}} x$. If $C$ is minimal in the Kazhdan-Lusztig right cell preorder, then $C$ is a lower set in the right p-cell preorder and we have

$$
\bigoplus_{x \in C} \mathbb{Z}\left[v, v^{-1}\right]^{p} \underline{H}_{x}=\bigoplus_{x \in C} \mathbb{Z}\left[v, v^{-1}\right] \underline{H}_{x}
$$


as $\mathbb{Z}\left[v, v^{-1}\right]$-submodules of $\mathcal{H}$.

Proof. Assume that ${ }^{p} \underline{H}_{y}$ occurs with non-zero coefficient in ${ }^{p} \underline{H}_{x} h$ for some $h \in \mathcal{H}$. Write ${ }^{p} \underline{H}_{x} h=$ $\left(\underline{H}_{x}+\sum_{z<x}^{p} m_{z, x} \underline{H}_{z}\right) h$. If $\underline{H}_{y}$ occurs with non-zero coefficient in the product $\underline{H}_{x} h$ then we have by definition $y \underset{R}{\stackrel{0}{\leqslant}} x$. If $\underline{H}_{y}$ occurs with non-zero coefficient in one of the products ${ }^{p} m_{z, x} \underline{H}_{z} h$, then we have ${ }^{p} m_{z, x} \neq 0$ and by Corollary 3.20 that $y \underset{R}{\leqslant} z \underset{R}{\leqslant} x$.

Denote by $C_{\min }$ the set of right-minimal elements in $C$. Our arguments above show that we have the following inclusions:

$$
\{\stackrel{p}{\underset{R}{\leftarrow}} C\}=\bigcup_{x \in C_{\min }}\{\stackrel{p}{\underset{R}{\leqslant}} x\} \subseteq \bigcup_{x \in C_{\min }}\{\underset{R}{\stackrel{0}{\leqslant}} x\}=\{\underset{R}{\stackrel{0}{\leqslant}} C\}
$$

The equalities on the left and right hand side follow from Lemma 3.18. In particular, $C$ is contained in the left hand side. Thus, if $C$ is minimal in the Kazhdan-Lusztig right cell preorder, we actually have equality which implies the claim as the left hand side obviously is a lower set in the $p$-cell preorder. In this case, Corollary 3.20 shows that for any $x \in C$ the $p$-canonical basis element ${ }^{p} \underline{H}_{x}$ can be written in terms of Kazhdan-Lusztig basis elements indexed by elements in $C$ which implies the statement about the span of the $p$-canonical and the Kazhdan-Lusztig basis elements.

Lemma 3.23. Let $C$ be a Kazhdan-Lusztig right cell. Assume that all Kazhdan-Lusztig right cells smaller or equal than $C$ in the Kazhdan-Lusztig right cell preorder satisfy the assumption of Corollary 3.20. Then $\{\underset{R}{\stackrel{0}{\leqslant}} C\}$ is a lower set in the right $p$-cell preorder and we have

$$
\bigoplus_{x \in\{\underset{R}{p} \leqslant C\}} \mathbb{Z}\left[v, v^{-1}\right]^{p} \underline{H}_{x}=\bigoplus_{\substack{0 \\ x \in\{\underset{R}{\leqslant} C\}}} \mathbb{Z}\left[v, v^{-1}\right] \underline{H}_{x}
$$

as $\mathbb{Z}\left[v, v^{-1}\right]$-submodules of $\mathcal{H}$. Moreover, $C$ decomposes as a union of right p-cells.

Proof. We proceed by induction on the height of $C$ in the Kazhdan-Lusztig right cell preorder. By assumption the height of any cell in the right cell preorder is finite.

Lemma 3.22 gives the induction start. Let $C$ be of height $\geqslant 2$. By induction for all predecessors of $C$ in the Kazhdan-Lusztig right cell preorder we know our claim holds for $\left\{{\underset{R}{<}}_{C}^{0}\right\}$. Therefore, we may pass to the quotient

$$
\mathcal{H} / \bigoplus_{x \in\left\{\stackrel{0}{R}_{R}^{0} C\right\}} \mathbb{Z}\left[v, v^{-1}\right] \underline{H}_{x}
$$

where $C$ becomes the smallest cell. Note that this quotient is a right $\mathcal{H}$-module that is free as a $\mathbb{Z}\left[v, v^{-1}\right]$-module and admits a $p$-canonical as well as a Kazhdan-Lusztig basis. Denote by

$$
\pi: \mathcal{H} \rightarrow \mathcal{H} / \bigoplus_{x \in\left\{\stackrel{0}{<}<_{R} C\right\}} \mathbb{Z}\left[v, v^{-1}\right] \underline{H}_{x}
$$

the projection to the quotient. Applying $\pi$ amounts to forgetting all basis elements indexed by elements in $\left\{\stackrel{0}{R}_{<}^{0} C\right\}$ when expressing an element in the Hecke algebra in the $p$-canonical or KazhdanLusztig basis. For this reason, the action of $\mathcal{H}$ on this right module captures a lot of information about the $p$-cell as well as the Kazhdan-Lusztig cell structure in the following sense: For $x, y \in\{\underset{R}{\nless} C$ C 
we have $y \underset{R}{\stackrel{p}{\leftarrow}} x$ if and only if there exists an element $h \in \mathcal{H}$ such that $\pi\left({ }^{p} \underline{H}_{y}\right)$ occurs with non-trivial coefficient in $\pi\left({ }^{p} \underline{H}_{x}\right) h$. A similar statement holds for the Kazhdan-Lusztig cell structure. This allows us to conclude as in the proof of Lemma 3.22 .

\section{4 (Counter-)Examples}

In this section, we will give some examples of the $p$-cell structure of finite Weyl groups. In this section we will only give the Dynkin diagram and consider the corresponding Cartan matrix as input. One may obtain a Kac-Moody root datum from any based root datum of the corresponding connected semi-simple algebraic group.

We will restrict to those examples that give counterexamples to obvious generalizations of known results from Kazhdan-Lusztig cell theory. All results in this section were obtained using computer calculations. Denote by $w_{0}$ the longest element in the corresponding finite Weyl group.

\subsubsection{Type $B_{2}$}

We label the simple reflections as follows:

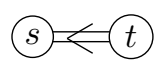

The following diagrams show the right (resp. two-sided) cells and the corresponding preorders in type $B_{2}$ :

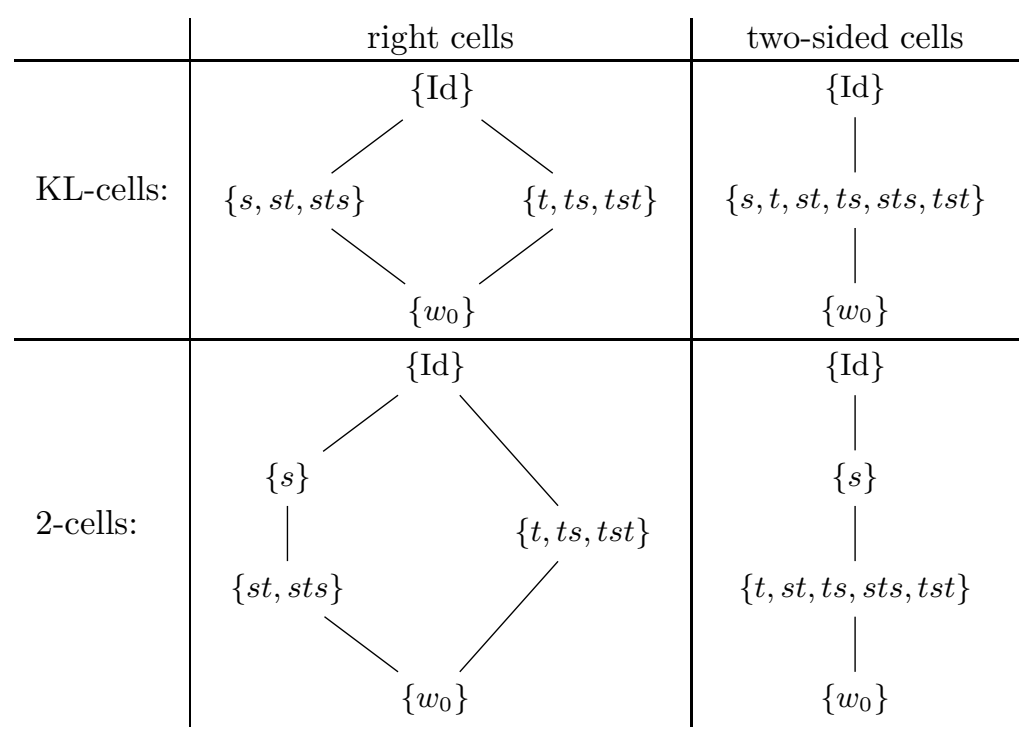

Lusztig showed in [Lus83, Proposition 3.8] that the set $C$ of non-trivial elements in a Coxeter group that have a unique reduced expression always forms a Kazhdan-Lusztig two-sided cell and for any $s \in S$ the set ${ }_{s} C:=\{w \in C \mid \mathcal{L}(w)=\{s\}\}$ gives a Kazhdan-Lusztig right cell. The example above shows that both statements do not hold for $p$-cells in general.

Observe that in characteristic 0 we have for $x, y \in W$ (see [Lus03, Corollary 11.7]) the following equivalences

$$
x \underset{R}{\stackrel{0}{\leqslant}} y \Leftrightarrow y w_{0} \underset{R}{\stackrel{0}{\leqslant}} x w_{0} \Leftrightarrow w_{0} y \underset{R}{\stackrel{0}{\leqslant}} w_{0} x
$$


and the same statement for the left and two-sided cell preorder. The example above also shows that the analogous statement does not hold for $p$-cells.

\subsubsection{Type $G_{2}$}

We label the simple reflections as follows:

\section{$s \approx t$}

The following diagrams show the right (resp. two-sided) cells and the corresponding preorders in type $G_{2}$ using the notation from the last subsection. In particular, $C,{ }_{s} C$ and ${ }_{t} C$ are defined as in Section 3.4.1.

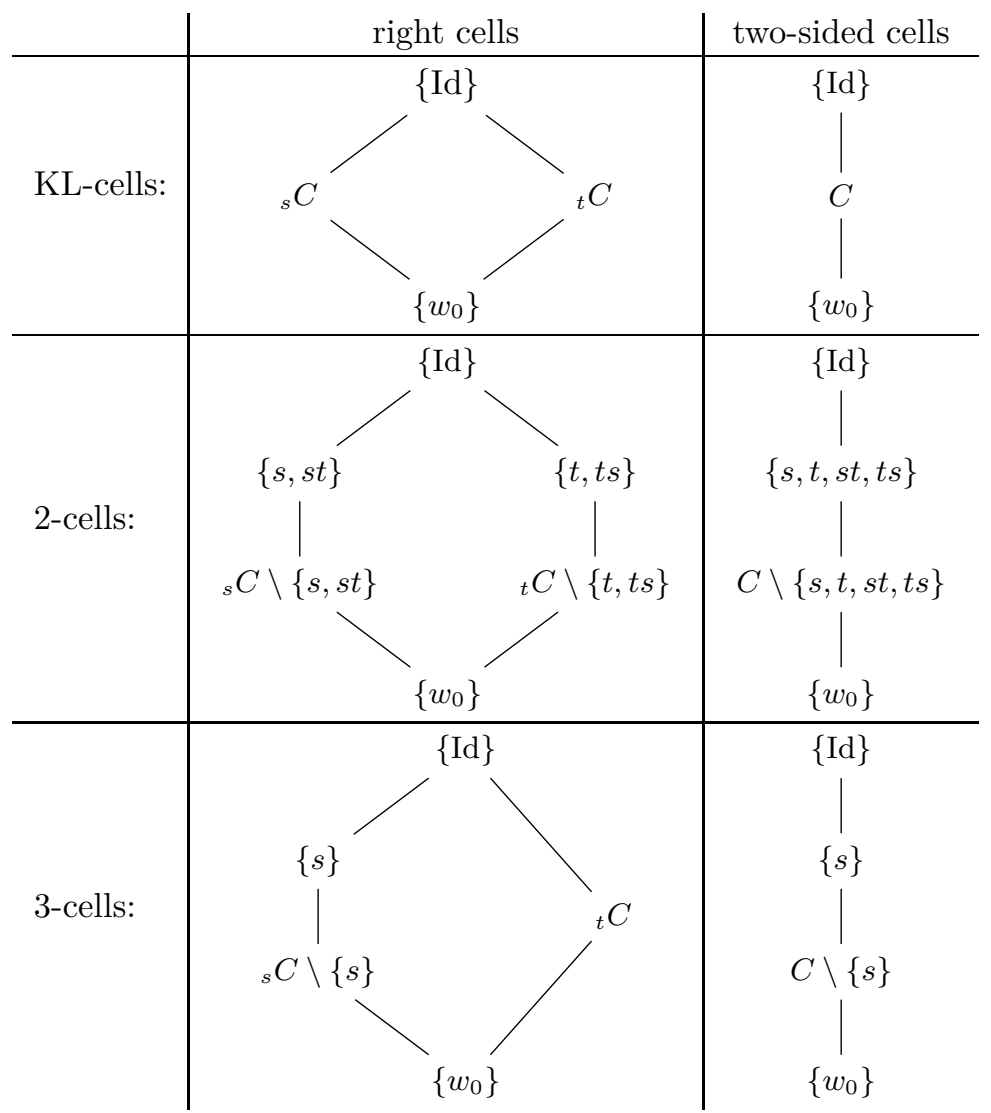

\subsubsection{Kazhdan-Lusztig cells do not decompose into $p$-cells}

In this section, we will present the smallest example where Kazhdan-Lusztig cells do not decompose into $p$-cells. This happens in type $C_{3}$ for $p=2$. We label the simple reflections as follows (also note their colours):

$$
\text { (1) } \Rightarrow 2 \Leftrightarrow \text { Cartan matrix: }\left(\begin{array}{ccc}
2 & -1 & 0 \\
-2 & 2 & -1 \\
0 & -1 & 2
\end{array}\right)
$$


Explicit computer calculation gives the following Kazhdan-Lusztig right cells:

$$
\begin{aligned}
C_{0} & =\{\mathrm{Id}\} \\
C_{1} & =\{1,12,121,123\} \\
C_{2} & =\{2,21,23,212,2123\} \\
C_{3} & =\{3,32,321,3212,32123\} \\
C_{4} & =\{13,132,1321\} \\
C_{5} & =\{213,2132,21321\} \\
C_{6} & =\{232,2321,23212\} \\
C_{7} & =\{2121,21213,212132,2121321,21213213\} \\
C_{8} & =\{1213,12132,121321\} \\
C_{9} & =\{1232,12321,123212\} \\
C_{10} & =\{13212,132123,1213212,1232123,12132123\} \\
C_{11} & =\{21232,212321,2123212\} \\
C_{12} & =\{232123,232121,2321213,23212132\} \\
C_{13} & =\left\{w_{0}\right\}
\end{aligned}
$$

For $p=2$ these right Kazhdan-Lusztig cells exhibit the following decomposition behaviour into right $p$-cells:

$$
\begin{aligned}
C_{2} & =\underbrace{\{2,21\}}_{p C_{2^{\prime}}} \cup \underbrace{}_{p C_{2^{\prime \prime}}\{23,212,2123\}} \\
C_{3} & =\underbrace{\{3,32\}}_{p C_{3^{\prime}}} \cup \underbrace{\{232\}}_{p C_{3^{\prime \prime}}\{321,3212,32123\}} \cup \underbrace{\{2321,23212,232123\}}_{p C_{6}} \cup \underbrace{}_{p C_{6 / 12}\{232121,2321213,23212132\}} \\
C_{6} \cup C_{12} & =\underbrace{\{23213\} \backslash\{2,3,6,12\}}_{p C_{12}}
\end{aligned}
$$

The Hasse-diagrams of the cell preorders look as follows. We display Kazhdan-Lusztig right cells on the left and right $p$-cells on the right. In these diagrams the cells that are depicted at one height 
form a two-sided cell.
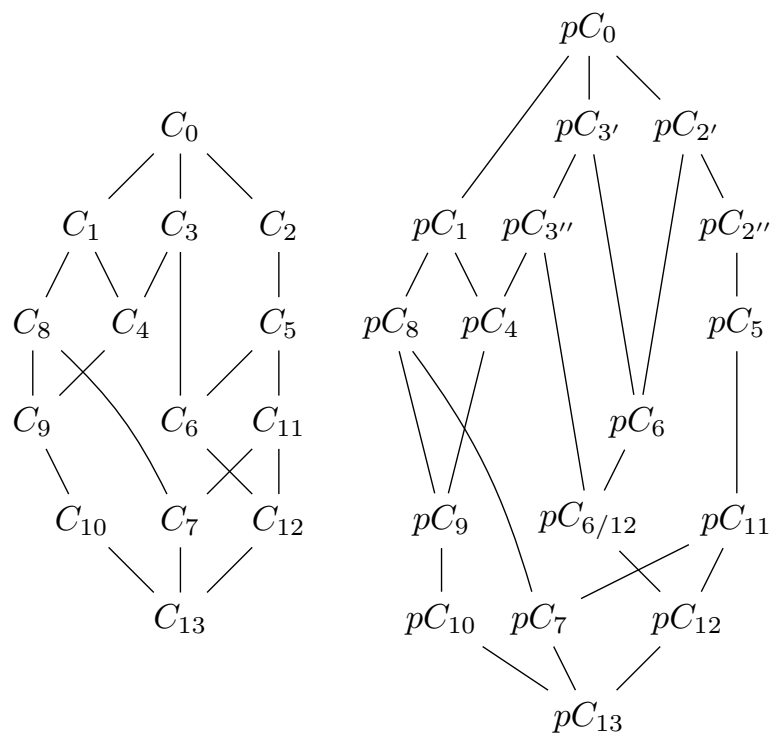

Finally, let us try to explain the non-trivial decomposition behaviour. For the elements in $C_{6} \cup C_{12}$ we have:

$$
\begin{aligned}
{ }^{2} \underline{H}_{23212} & =\underline{H}_{23212}+\underline{H}_{232} \\
{ }^{2} \underline{H}_{232123} & =\underline{H}_{232123}+\left(v+v^{-1}\right) \underline{H}_{232} \\
{ }^{2} \underline{H}_{23212132} & =\underline{H}_{23212132}+\underline{H}_{232123} \\
{ }^{2} \underline{H}_{x} & =\underline{H}_{x} \text { for } x \in\left(C_{6} \cup C_{12}\right) \backslash\{23212,232123,23212132\}
\end{aligned}
$$

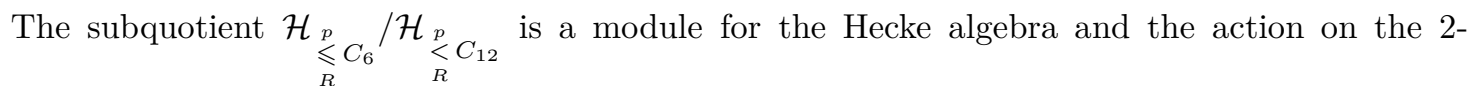
canonical basis of this module can be encoded in the following graph:

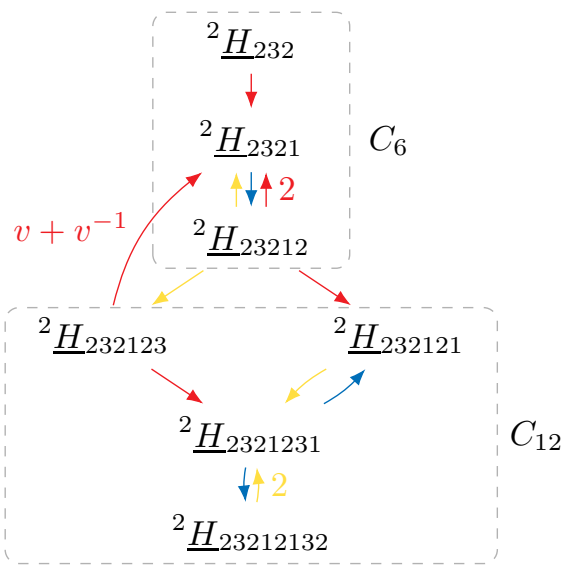

Note that we omitted all edge labels equal to 1 and all loops labelled with $v+v^{-1}$. The strongly connected components of this graph give the right $p$-cells $p C_{6}, p C_{6 / 12}$ and $p C_{12}$. From this we see that neither two-sided nor right Kazhdan-Lusztig cells decompose into the corresponding $p$-cells in this example. 
In this case we cannot apply the decomposition criterion from Section 3.3 because the KazhdanLusztig right cell $C_{12}$ does not satisfy the assumptions of Corollary 3.20.

In type $B_{3}$ the right (and two-sided) Kazhdan-Lusztig cells do decompose into right (resp. twosided) $p$-cells, whereas in type $B_{4}$ they do not. This calculation together with Proposition 3.24 below and Corollary 4.26 completely settles the question of when right Kazhdan-Lusztig cells decompose into right $p$-cells in types $B$ and $C$. In summary, right Kazhdan-Lusztig cells in types $B_{n}$ and $C_{n}$ decompose into right $p$-cells for $p>2$ or $p=2$ and $n \leqslant 3$ in type $B_{n}$.

Proposition 3.24. Suppose the Kazhdan-Lusztig right cells of $H$ do not decompose into right $p$-cells for some $p>0$. Let $W$ be a crystallographic Coxeter group that contains $H$ as standard parabolic subgroup. Then the Kazhdan-Lusztig right cells of $W$ do not decompose into p-cells.

Proof. By assumption we may find two distinct right Kazhdan-Lusztig cells $C_{1}, C_{2} \subseteq H$ and a right $p$-cell ${ }^{p} C \subseteq H$ for some $p$ such that ${ }^{p} C \cap C_{1}$ and ${ }^{p} C \cap C_{2}$ are non-empty. By Theorem 3.9 we may find right Kazhdan-Lusztig cells $\widetilde{C_{1}}, \widetilde{C_{2}} \subseteq W$ and a right $p$-cell $\widetilde{{ }^{p} C} \subseteq W$ such that $C_{i} \subseteq \widetilde{C_{i}}$ for $i \in\{1,2\}$ and ${ }^{p} C \subseteq \widetilde{{ }^{p} C}$.

Observe that for $i \in\{1,2\}$ the set $\widetilde{{ }^{p} C} \cap \widetilde{C_{i}}$ is non-empty as it contains ${ }^{p} C \cap C_{i}$. Therefore, it is enough to show that $\widetilde{C_{1}}$ and $\widetilde{C_{2}}$ are disjoint. Let $X$ be the set of representatives of minimal length of cosets in $H \backslash W$. To conclude we need to recall a result about the induction of Kazhdan-Lusztig cells (see [Gec03, Theorem 1] for the version for left cells):

Theorem 3.25 (Induction of right Kazhdan-Lusztig cells). Let $H \subseteq W$ be a standard parabolic subgroup, and let $C$ be a right Kazhdan-Lusztig cell of $H$. Then $C \cdot X \overline{\text { is a }}$ union of right cells of $W$.

Since we have $\widetilde{C_{i}} \subseteq C_{i} \cdot X$ for $i \in\{1,2\}$, the claim follows from the fact that $C_{1} \cdot X$ and $C_{2} \cdot X$ are disjoint.

\section{Left and Right Star Operations}

\subsection{Definition and Numerical Consequences}

In the section, we will prove consequences of the Kazhdan-Lusztig star-operations for the $p$-canonical basis. The star-operations were originally introduced in [KL79, §4], generalizing (dual) Knuth operations from the symmetric group to pairs of simple reflections $r, t \in S$ in general Coxeter groups with $m_{r, t}=3$. In the literature, there does not seem to exist a consensus on how to generalize the star-operations to the case $3<m_{r, t}<\infty$. We propose the following generalization as in [BG15, Remark 4.3]:

Definition 4.1. Let $r, t \in S$ be two simple reflections. Define:

$$
\begin{aligned}
& \mathcal{D}_{L}(r, t):=\{w \in W|| \mathcal{L}(w) \cap\{r, t\} \mid=1\} \\
& \mathcal{D}_{R}(r, t):=\{w \in W|| \mathcal{R}(w) \cap\{r, t\} \mid=1\}
\end{aligned}
$$

Set $m:=m_{r, t}$. For $1 \leqslant k \leqslant m$ denote by ${ }_{r} \hat{k}=r t r t \ldots$ the alternating word in $r$ and $t$ starting in $r$ of length $k$. Recall that $W^{\{r, t\}}$ denotes the set of representatives of minimal length of cosets in $W /\langle r, t\rangle$ (see Definition 3.8). Any coset in $W /\langle r, t\rangle$ contains a unique element $\widetilde{w} \in W^{\{r, t\}}$ and can be partitioned in the following sets:

$$
\begin{cases}\{\widetilde{w}\} \cup\left\{\widetilde{w} \cdot{ }_{r} \hat{k} \mid 1 \leqslant k<m\right\} \cup\left\{\widetilde{w} \cdot{ }_{t} \hat{k} \mid 1 \leqslant k<m\right\} \cup\left\{\widetilde{w} \cdot{ }_{t} \hat{m}\right\} & \text { if } m<\infty, \\ \{\widetilde{w}\} \cup\left\{\widetilde{w} \cdot{ }_{r} \hat{k} \mid 1 \leqslant k\right\} \cup\left\{\widetilde{w} \cdot{ }_{t} \hat{k} \mid 1 \leqslant k\right\} & \text { if } m=\infty .\end{cases}
$$

For $m<\infty$ the element $\widetilde{w} \cdot{ }_{t} \hat{m}$ is the unique element of maximal length in the coset. The set $\left\{\widetilde{w} \cdot{ }_{x} \hat{k} \mid 1 \leqslant k<m\right\}$ for some $x \in\{r, t\}$ is called a right $\langle r, t\rangle$-string (also for $m=\infty$ ) and contained in $\mathcal{D}_{R}(r, t)$. The element $\widetilde{w} \cdot{ }_{x} \hat{k}$ is the $k$-th element in this string. 
It is easy to see that actually any element $w \in \mathcal{D}_{R}(r, t)$ lies in a right $\langle r, t\rangle$-string and can thus be written as $\widetilde{w} \cdot{ }_{x} \hat{k}$ for some $x \in\{r, t\}$ and $1 \leqslant k<m$ where $\widetilde{w}$ is the element of minimal length in the right coset $w\langle r, t\rangle \in W /\langle r, t\rangle$.

Assume $3 \leqslant m<\infty$. Then the right star operation $(-)^{*}$ is an involution on $\mathcal{D}_{R}(r, t)$ sending $w=\widetilde{w} \cdot{ }_{x} \hat{k}$ for $x$ and $k$ as above to $\widetilde{w} \cdot{ }_{x}(\widehat{m-k})$. The left star operation ${ }^{*}(-)$ is an involution on $\mathcal{D}_{L}(r, t)$ defined analogously.

It follows immediately that the left and right star operations are related via:

$$
{ }^{*} w=\left(\left(w^{-1}\right)^{*}\right)^{-1} .
$$

Fix for the rest of the section two simple reflections $r, t \in S$ with $3 \leqslant m:=m_{r, t}<\infty$. The multiplication formula for the Kazhdan-Lusztig basis does not easily generalize to the $p$-canonical basis. However it will still be important to understand the structure coefficients of the $p$-canonical basis a little bit better. The next lemma states a crucial observation that will be used frequently below.

Lemma 4.2. Let $x, z \in \mathcal{D}_{R}(r, t)$ with $r \in \mathcal{R}(x)$. The coefficient of $\underline{H}_{z}$ in ${ }^{p} \underline{H}_{x} \underline{H}_{t}$ is given by

$$
\delta_{z r \in \mathcal{D}_{R}(r, t)}^{p} m_{z r, x}+\delta_{z t \in \mathcal{D}_{R}(r, t)}^{p} m_{z t, x}
$$

where $\delta_{z r \in \mathcal{D}_{R}(r, t)}$ is the Kronecker delta.

Proof. Rewrite the product ${ }^{p} \underline{H}_{x} \underline{H}_{t}$ as follows:

$$
\begin{aligned}
{ }^{p} \underline{H}_{x} \underline{H}_{t}= & \left(\underline{H}_{x}+\sum_{w<x}{ }^{p} m_{w, x} \underline{H}_{w}\right) \underline{H}_{t} \\
= & \underline{H}_{x t}+\sum_{\substack{z \leqslant x \\
z t<z}} \mu(z, x) \underline{H}_{z}+\sum_{\substack{w<x \\
w t>w}} p m_{w, x}\left(\underline{H}_{w t}+\sum_{\substack{z \leqslant w \\
z t<z}} \mu(z, w) \underline{H}_{z}\right) \\
& +\sum_{\substack{z<x \\
z t<z}}\left(v+v^{-1}\right)^{p} m_{z, x} \underline{H}_{z}
\end{aligned}
$$

If $t$ is not in the right descent set of $z$, the coefficient in front of $\underline{H}_{z}$ has to be zero. By Proposition $2.5(\mathrm{v})$ the formula stated above also gives zero in this case. Thus, we assume $t \in \mathcal{R}(z)$ from now on. Since $z$ lies in $\mathcal{D}_{R}(r, t)$, this implies that $r$ does not lie in the right descent set of $z$. Consider an element $w \in W$ with ${ }^{p} m_{w, x} \neq 0$ such that $\underline{H}_{z}$ occurs with non-zero coefficient in $\underline{H}_{w} \underline{H}_{t}$. Observe that $w$ could be $x$. By Proposition 2.5(v) ${ }^{p} m_{w, x} \neq 0$ implies $r \in \mathcal{R}(x) \subseteq \mathcal{R}(w)$. Since the right descent sets differ, $w$ and $z$ cannot coincide. In particular, $w$ does not have $t$ in its right descent set and thus also lies in $\mathcal{D}_{R}(r, t)$. (Otherwise $\underline{H}_{z}$ could not occur with non-zero coefficient in $\underline{H}_{w} \underline{H}_{t}$.) Recall the following important fact about the $\mu$-coefficients (from [KL79, (2.3.f)]):

Lemma 4.3. Let $z<w \in W$ and $r \in \mathcal{R}(w) \backslash \mathcal{R}(z)$. Then we have:

$$
\mu(z, w) \neq 0 \Leftrightarrow w=z r
$$

Moreover, $\mu(z, w)=1$ in this case.

If $z<w$ holds, then we may apply this lemma to $z<w$ and the simple reflection $r$ to get that $w=z r$. Otherwise, we have $z=w t>w$ (due to the multiplication formula from [KL79, (2.3.b)]) and again $\mu(w, z)=1$. In both cases, we see that $z$ and $w$ lie in the same right $\langle r, t\rangle$-string and the coefficient of $\underline{H}_{z}$ in ${ }^{p} \underline{H}_{x} \underline{H}_{t}$ is

$$
\delta_{z r \in \mathcal{D}_{R}(r, t)}^{p} m_{z r, x}+\delta_{z t \in \mathcal{D}_{R}(r, t)}^{p} m_{z t, x} .
$$


Definition 4.4. The weak right Bruhat graph of $(W, S)$ is the labelled, directed graph with vertex set $W$ and edge set

$$
\{(w, w s) \mid w \in W, s \in S \backslash \mathcal{R}(w)\} .
$$

For $w \in W$ and $s \in S \backslash \mathcal{R}(w)$ the edge $(w, w s)$ is labelled by $\alpha_{s}$.

The reader may picture the formula from Lemma 4.2 as follows: Consider the subgraph of the weak right Bruhat graph on the vertices $\mathcal{D}_{R}(r, t) \cap(z\langle r, t\rangle)$ and only edges labelled by $\alpha_{r}$ or $\alpha_{t}$. In order to get the coefficient of $\underline{H}_{z}$ in ${ }^{p} \underline{H}_{x} \underline{H}_{t}$ we simply have to slide the coefficients ${ }^{p} m_{?, x}$ up along an edge labelled by $\alpha_{t}$ and down along an edge labelled by $\alpha_{r}$ and sum them up if two coefficients collide at a vertex in the process. Here, up and down are meant with respect to the weak right Bruhat order.

For the rest of the section, we will assume:

$$
p> \begin{cases}1 & \text { if } m=3 \\ 2 & \text { if } m=4 \\ 3 & \text { if } m=6\end{cases}
$$

This ensures that for $w \in\langle r, t\rangle$ we have ${ }^{p} \underline{H}_{w}=\underline{H}_{w}$ (see [JW17, §5.1, §5.2 and §5.6]) and thus Kazhdan-Lusztig cells and $p$-cells in $\langle r, t\rangle$ coincide.

Proposition 4.5. Let $\sigma_{x}=\left\{x_{1}<x_{2}<\ldots\right\}$ and $\sigma_{z}=\left\{z_{1}<z_{2}<\ldots\right\}$ be two right $\langle r, t\rangle$-strings consisting of $m-1$ elements. Then we have the following relations among the coefficients ${ }^{p} m_{z_{j}, x_{i}}$ :

$$
\begin{aligned}
& m=3 \Rightarrow\left\{\begin{array}{l}
{ }^{p} m_{z_{1}, x_{1}}={ }^{p} m_{z_{2}, x_{2}} \\
{ }^{p} m_{z_{2}, x_{1}}={ }^{p} m_{z_{1}, x_{2}}
\end{array}\right. \\
& m=4 \Rightarrow\left\{\begin{array}{l}
{ }^{p} m_{z_{1}, x_{1}}={ }^{p} m_{z_{3}, x_{3}} \\
{ }^{p} m_{z_{2}, x_{1}}={ }^{p} m_{z_{1}, x_{2}}={ }^{p} m_{z_{3}, x_{2}}={ }^{p} m_{z_{2}, x_{3}} \\
{ }^{p} m_{z_{3}, x_{1}}={ }^{p} m_{z_{1}, x_{3}} \\
{ }^{p} m_{z_{2}, x_{2}}={ }^{p} m_{z_{1}, x_{1}}+{ }^{p} m_{z_{3}, x_{1}}
\end{array}\right. \\
& m=6 \Rightarrow\left\{\begin{array}{l}
{ }^{p} m_{z_{1}, x_{1}}={ }^{p} m_{z_{5}, x_{5}} \\
{ }^{p} m_{z_{2}, x_{1}}={ }^{p} m_{z_{1}, x_{2}}={ }^{p} m_{z_{5}, x_{4}}={ }^{p} m_{z_{4}, x_{5}} \\
{ }^{p} m_{z_{3}, x_{1}}={ }^{p} m_{z_{1}, x_{3}}={ }^{p} m_{z_{5}, x_{3}}={ }^{p} m_{z_{3}, x_{5}} \\
{ }^{p} m_{z_{4}, x_{1}}={ }^{p} m_{z_{1}, x_{4}}={ }^{p} m_{z_{5}, x_{2}}={ }^{p} m_{z_{2}, x_{5}} \\
{ }^{p} m_{z_{5}, x_{1}}={ }^{p} m_{z_{1}, x_{5}} \\
{ }^{p} m_{z_{2}, x_{2}}={ }^{p} m_{z_{4}, x_{4}}={ }^{p} m_{z_{1}, x_{1}}+{ }^{p} m_{z_{3}, x_{1}} \\
{ }^{p} m_{z_{3}, x_{2}}={ }^{p} m_{z_{2}, x_{3}}={ }^{p} m_{z_{4}, x_{3}}={ }^{p} m_{z_{3}, x_{4}}={ }^{p} m_{z_{2}, x_{1}}+{ }^{p} m_{z_{4}, x_{1}} \\
{ }^{p} m_{z_{4}, x_{2}}={ }^{p} m_{z_{2}, x_{4}}={ }^{p} m_{z_{3}, x_{1}}+{ }^{p} m_{z_{5}, x_{1}} \\
{ }^{p} m_{z_{3}, x_{3}}={ }^{p} m_{z_{1}, x_{1}}+{ }^{p} m_{z_{3}, x_{1}}+{ }^{p} m_{z_{5}, x_{1}}
\end{array}\right.
\end{aligned}
$$

Proof. Comparing Laurent polynomials coefficient-wise induces a partial order which we will use in the following. For $x \in \sigma_{x}$ with $r \in \mathcal{R}(x)$, rewrite ${ }^{p} \underline{H}_{x} \underline{H}_{t}$ in terms of the $p$-canonical basis to get:

$$
\sum_{v \leqslant x t}{ }^{p} \mu_{x, t}^{v} \underline{H}_{v}
$$

Express this in the Kazhdan-Lusztig basis and use Lemma 4.2 to see that we have the following inequality for $z \in \sigma_{z}$ which is actually satisfied with equality:

$$
\sum_{z \leqslant v \leqslant x t}{ }^{p} \mu_{x, t}^{v}{ }^{p} m_{z, v} \leqslant \delta_{z r \in \mathcal{D}_{R}(r, t)}^{p} m_{z r, x}+\delta_{z t \in \mathcal{D}_{R}(r, t)}^{p} m_{z t, x}
$$


We want to use a weaker form of this inequality together with the fact that we understand the structure coefficients in the right $\langle r, t\rangle$-coset of $x$ (see Corollary 3.13). Let $s_{i} \in\{r, t\}$ be the simple reflection such that $x_{i} s_{i}>x_{i}$ for $1 \leqslant i \leqslant m-1$. Write $x_{0}$ (resp. $x_{m}$ ) for the shortest (resp. longest) element in the right $\langle r, t\rangle$-coset of $x$. Similarly for $z_{0}$ and $z_{m}$. We can restrict the sum on the left hand side of (4) to $v \in \sigma_{x}$ and $v \leqslant x t$. From Corollary 3.13 and the explicit knowledge of the structure constants of the Kazhdan-Lusztig basis in the dihedral case we deduce:

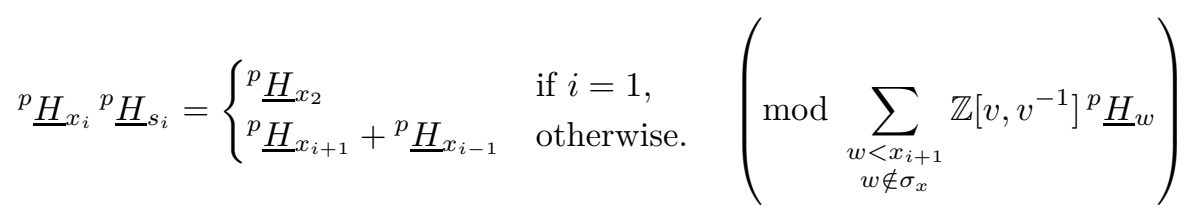

Using this in inequality (4) and letting $x$ and $z$ in their right $\langle r, t\rangle$-string vary, we obtain for $1 \leqslant$ $i, j \leqslant m-1$ :

$$
\delta_{x_{i+1} \in \sigma_{x}}^{p} m_{z_{j}, x_{i+1}}+\delta_{x_{i-1} \in \sigma_{x}}^{p} m_{z_{j}, x_{i-1}} \leqslant \delta_{z_{j+1} \in \sigma_{z}}^{p} m_{z_{j+1}, x_{i}}+\delta_{z_{j-1} \in \sigma_{z}}^{p} m_{z_{j-1}, x_{i}} \quad\left(*_{i, j}\right)
$$

Surprisingly enough, any solution to this system of inequalities satisfies all inequalities with equality. We will solve this system of linear inequalities for $m=6$ and leave the cases $m \in\{3,4\}$ to the reader. To simplify notation, write $a_{j, i}={ }^{p} m_{z_{j}, x_{i}}$ for all $1 \leqslant i, j \leqslant m-1$ and view them as indeterminates. The set of inequalities can be partitioned into two sets of inequalities which can be solved completely independently:

$$
\left\{\left(*_{i, j}\right) \mid i+j \text { even }\right\} \cup\left\{\left(*_{i, j}\right) \mid i+j \text { odd }\right\}
$$

First, let us consider $\left\{\left(*_{i, j}\right) \mid i+j\right.$ even $\}$ :

$$
\begin{aligned}
a_{1,2} & \leqslant a_{2,1} \\
a_{3,2} & \leqslant a_{2,1}+a_{4,1} \\
a_{5,2} & \leqslant a_{4,1} \\
a_{2,3}+a_{2,1} & \leqslant a_{1,2}+a_{3,2} \\
a_{4,3}+a_{4,1} & \leqslant a_{3,2}+a_{5,2} \\
a_{1,4}+a_{1,2} & \leqslant a_{2,3} \\
a_{3,4}+a_{3,2} & \leqslant a_{2,3}+a_{4,3} \\
a_{5,4}+a_{5,2} & \leqslant a_{4,3} \\
a_{2,5}+a_{2,3} & \leqslant a_{1,4}+a_{3,4} \\
a_{4,5}+a_{4,3} & \leqslant a_{3,4}+a_{5,4} \\
a_{1,4} & \leqslant a_{2,5} \\
a_{3,4} & \leqslant a_{2,5}+a_{4,5} \\
a_{5,4} & \leqslant a_{4,5}
\end{aligned}
$$

Consider the following chain of inequalities:

$$
\stackrel{\text { (iv) }}{\leqslant} a_{1,2}+a_{3,2}-a_{2,3} \stackrel{\text { (vii) }}{\leqslant} a_{1,2}+a_{4,3}-a_{3,4} \stackrel{(\mathrm{x})}{\leqslant} a_{1,2}+a_{5,4}-a_{4,5} \stackrel{\text { (xiii) }}{\leqslant} a_{1,2} \stackrel{\text { (i) }}{\leqslant} a_{2,1}
$$

This implies that the inequalities (i), (iv), (vii), (x) and (xiii) are all satisfied with equality, which in turn implies:

$$
a_{2,3}=a_{3,2} \quad a_{3,4}=a_{4,3}
$$

Next, consider the following chain:

$$
a_{4,1} \stackrel{(\mathrm{v})}{\leqslant} a_{3,2}+a_{5,2}-a_{4,3} \stackrel{(\text { vii })}{=} a_{2,3}-a_{3,4}+a_{5,2} \stackrel{(\mathrm{ix})}{\leqslant} a_{1,4}-a_{2,5}+a_{5,2} \stackrel{(\mathrm{xi})}{\leqslant} a_{5,2} \stackrel{\text { (iii) }}{\leqslant} a_{4,1}
$$


This shows that the inequalities (iii), (v), (ix) and (xi) are also satisfied with equality, from which we deduce:

$$
a_{2,3}=a_{3,2}=a_{3,4}=a_{4,3}
$$

Finally, we have

$$
a_{1,4}+a_{1,2} \stackrel{(\text { vi })}{\leqslant} a_{2,3}=a_{3,2} \stackrel{(\text { ii })}{\leqslant} a_{2,1}+a_{4,1}
$$

and

$$
a_{5,4}+a_{5,2} \stackrel{\text { (viii) }}{\leqslant} a_{4,3}=a_{3,4} \stackrel{\text { (xii) }}{\leqslant} a_{2,5}+a_{4,5}
$$

which imply using $a_{1,2}=a_{2,1}$ and $a_{5,4}=a_{4,5}$ respectively

$$
a_{1,4} \leqslant a_{4,1} \quad a_{5,2} \leqslant a_{2,5}
$$

Using $a_{1,4}=a_{2,5}$ and $a_{4,1}=a_{5,2}$ finishes the argument.

Next, we solve $\left\{\left(*_{i, j}\right) \mid i+j\right.$ odd $\}$ :

$$
\begin{aligned}
a_{2,2} & \leqslant a_{1,1}+a_{3,1} \\
a_{4,2} & \leqslant a_{3,1}+a_{5,1} \\
a_{1,3}+a_{1,1} & \leqslant a_{2,2} \\
a_{3,3}+a_{3,1} & \leqslant a_{2,2}+a_{4,2} \\
a_{5,3}+a_{5,1} & \leqslant a_{4,2} \\
a_{2,4}+a_{2,2} & \leqslant a_{1,3}+a_{3,3} \\
a_{4,4}+a_{4,2} & \leqslant a_{3,3}+a_{5,3} \\
a_{1,5}+a_{1,3} & \leqslant a_{2,4} \\
a_{3,5}+a_{3,3} & \leqslant a_{2,4}+a_{4,4} \\
a_{5,5}+a_{5,3} & \leqslant a_{4,4} \\
a_{2,4} & \leqslant a_{1,5}+a_{3,5} \\
a_{4,4} & \leqslant a_{3,5}+a_{5,5}
\end{aligned}
$$

In this case we argue as follows:

$$
a_{1,1} \stackrel{\left(\mathrm{iii}^{\prime}\right)}{\leqslant} a_{2,2}-a_{1,3} \stackrel{(\text { (vi') }}{\leqslant} a_{3,3}-a_{2,4} \stackrel{\left(\mathrm{ix}^{\prime}\right)}{\leqslant} a_{4,4}-a_{3,5} \stackrel{(\text { (xii') }}{\leqslant} a_{5,5}
$$

We use this in the last inequality of the following chain:

$a_{3,1} \stackrel{(\text { (iv') }}{\leqslant} a_{2,2}+a_{4,2}-a_{3,3} \stackrel{\text { (vii') }}{\leqslant} a_{2,2}+a_{5,3}-a_{4,4} \stackrel{\left(\mathrm{x}^{\prime}\right)}{\leqslant} a_{2,2}-a_{5,5} \stackrel{\left(\mathrm{i}^{\prime}\right)}{\leqslant} a_{1,1}+a_{3,1}-a_{5,5} \leqslant a_{3,1}$ This implies that $a_{1,1}=a_{5,5}$ and the inequalities (i'), (iii'), (iv'), (vi'), (vii'), (ix'), (x') and (xii') are satisfied with equality. Moreover, we have the following equivalences:

$$
\begin{aligned}
\left(\mathrm{i}^{\prime}\right)=\left(\mathrm{iii}^{\prime}\right) & \Leftrightarrow a_{1,3}=a_{3,1} \Leftrightarrow\left(\mathrm{iv}^{\prime}\right)=\left(\mathrm{vi}^{\prime}\right) \\
& \Leftrightarrow a_{2,4}=a_{4,2} \Leftrightarrow\left(\mathrm{vii}^{\prime}\right)=\left(\mathrm{ix}^{\prime}\right) \\
& \Leftrightarrow a_{5,3}=a_{3,5} \Leftrightarrow\left(\mathrm{x}^{\prime}\right)=\left(\mathrm{xii}^{\prime}\right)
\end{aligned}
$$

The last four inequalities can be used as follows:

$$
\begin{aligned}
& a_{5,3}+a_{5,1} \stackrel{\left(\mathrm{v}^{\prime}\right)}{\leqslant} a_{4,2} \stackrel{\left(\mathrm{ii}^{\prime}\right)}{\leqslant} a_{3,1}+a_{5,1} \\
& a_{1,5}+a_{1,3} \stackrel{\text { (viii') }}{\leqslant} a_{2,4} \stackrel{\left(\text { xi' }^{\prime}\right)}{\leqslant} a_{1,5}+a_{3,5}
\end{aligned}
$$


This gives $a_{5,3} \leqslant a_{3,1}=a_{1,3} \leqslant a_{3,5}=a_{5,3}$ and finishes the argument.

Finally, observe that the space of solutions for these (in)equalities is a free $\mathbb{Z}\left[v, v^{-1}\right]$-module of rank $m-1$ and we can choose $\left\{a_{1,1}, a_{2,1}, \ldots, a_{m-1,1}\right\}$ as a basis. In other words, the solution is uniquely determined after fixing these Laurent polynomials. From all these equalities, the reader can easily deduce the relations given in the proposition where we expressed each coefficient in terms of our chosen basis of the solution space.

Remark 4.6. In the proof Proposition 4.5 we have introduced a set of (in)equalities governing the base change coefficients between two $\langle r, t\rangle$-strings $\sigma_{x}$ and $\sigma_{z}$ and shown that its of space of solutions is a free $\mathbb{Z}\left[v, v^{-1}\right]$-module of rank $m-1$. Of course, not every solution gives a possible set of base change coefficients $\left\{{ }^{p} m_{z_{j}, x_{i}} \mid 1 \leqslant i, j \leqslant m-1\right\}$ as these coefficients have to satisfy more constraints: Proposition 2.5(iii) shows that these coefficients are self-dual and have non-negative integers as coefficients. Moreover, due to Proposition 2.5(v) these coefficients satisfy parity vanishing for fixed $i$ and arbitrary $1 \leqslant j \leqslant m-1$. This is also the underlying reason why we could partition the set of inequalities in two sets.

Corollary 4.7. For $z \leqslant x \in \mathcal{D}_{R}(r, t)$ one has:

$$
{ }^{p} m_{z, x}={ }^{p} m_{z^{*}, x^{*}}
$$

Proof. Note that ${ }^{p} m_{z, x}={ }^{p} m_{z^{*}, x^{*}}$ asks only for $a_{i, j}=a_{m-i, m-j}$ (in the notation of the proof of Proposition 4.5) for $1 \leqslant i, j \leqslant m-1$ whereas we have shown many more relations among these coefficients in the last proposition.

In the proof of Proposition 4.5 we have seen that when translating ${ }^{k} B_{x}$ by ${ }^{k} B_{t}$ for $x \in \mathcal{D}_{R}(r, t)$ with $x t>x$ the available coefficient of $\underline{H}_{z}$ in ${ }^{p} \underline{H}_{x} \underline{H}_{t}$ for $z \in \mathcal{D}_{R}(r, t)$ is completely subsumed by the neighbouring elements of $x$ in its right $\langle r, t\rangle$-string. This implies the following about the structure coefficients:

Corollary 4.8. Let $x, z \in \mathcal{D}_{R}(r, t)$ with $x t>x$. Then ${ }^{p} \mu_{x, t}^{z}$ vanishes unless $x$ and $z$ are neighbouring elements in the same right $\langle r, t\rangle$-string.

The next result will allow us to apply the star-operations to the study of $p$-cells. It is a generalization of [Lus85, (10.4.1), (10.4.2) and (10.4.3)]:

Proposition 4.9. Let $\sigma_{x}=\left\{x_{1}<x_{2}<\ldots\right\}$ (resp. $\left.\sigma_{z}=\left\{z_{1}<z_{2}<\ldots\right\}\right)$ be two right $\langle r, t\rangle$-strings consisting of $m-1$ elements. For any $s \in S \backslash \mathcal{L}\left(x_{1}\right)$ all the relations stated in Proposition 4.5 hold with ${ }^{p} m_{z_{j}, x_{i}}$ replaced by ${ }^{p} \mu_{s, x_{i}}^{z_{j}}$.

Proof. Lemma 4.11 below shows that all elements in $\sigma_{x}$ lie in the same right $p$-cell. We therefore deduce from Lemma 3.4 that all elements in $\sigma_{x}$ have the same left descent set and satisfy $s x_{i}>x_{i}$ for $1 \leqslant i \leqslant m-1$.

Write $x_{0}$ (resp. $x_{m}$ ) for the shortest (resp. longest) element in the right $\langle r, t\rangle$-coset of $x$. Similarly for $z_{0}$ and $z_{m}$. We are interested in the structure coefficients ${ }^{p} \mu_{s, x_{i}}^{z_{j}}$ for $1 \leqslant i, j \leqslant m-1$. To simplify notation, write $a_{j, i}={ }^{p} \mu_{s, x_{i}}^{z_{j}}$.

Fix $1 \leqslant i \leqslant m-1$ arbitrary. We may assume without loss of generality $x_{i} t>x_{i}$ and thus $r \in \mathcal{R}\left(x_{i}\right)$. The main idea is to express ${ }^{p} \underline{H}_{s}{ }^{p} \underline{H}_{x_{i}}{ }^{p} \underline{H}_{t}$ in the $p$-canonical basis in different ways and to analyze the coefficients in front of basis elements indexed by elements in $\sigma_{z}$. Depending on which multiplication in ${ }^{p} \underline{H}_{s}{ }^{p} \underline{H}_{x_{i}}{ }^{p} \underline{H}_{t}$ we first carry out, we get two ways to express this product in terms of the $p$-canonical basis. On the one hand we have:

$$
\begin{aligned}
{ }^{p} \underline{H}_{s}{ }^{p} \underline{H}_{x_{i}}{ }^{p} \underline{H}_{t} & =\left(\sum_{u \leqslant s x_{i}}{ }^{p} \mu_{s, x_{i}}^{u} \underline{H}_{u}\right)^{p} \underline{H}_{t} \\
& =\sum_{\substack{u \leqslant s x_{i} \\
u t<u}}\left(v+v^{-1}\right)^{p} \mu_{s, x_{i}}^{u} \underline{H}_{u}+\sum_{\substack{u \leqslant s x_{i} \wedge w \leqslant u t \\
u t>u}}{ }^{p} \mu_{s, x_{i}}^{u}{ }^{p} \mu_{u, t}^{w} \underline{H}_{w}
\end{aligned}
$$


Consider an element $u \leqslant s x_{i}$ with ${ }^{p} \mu_{s, x_{i}}^{u} \neq 0$. The version of Lemma 3.4 for left cells implies $r \in \mathcal{R}(u)$ and in particular $u$ is not minimal in its right $\langle r, t\rangle$-coset. This implies:

$$
u \in \mathcal{D}_{R}(r, t) \Leftrightarrow u t>u
$$

Assume $u \in \mathcal{D}_{R}(r, t)$ (otherwise ${ }^{p} \underline{H}_{u}{ }^{p} \underline{H}_{t}=\left(v+v^{-1}\right)^{p} \underline{H}_{u}$ does not contain any $p$-canonical basis elements indexed by elements in $\sigma_{z}$ ). Combining Corollary 4.8 and Corollary 3.13 we have full control over the $p$-canonical basis elements indexed by elements in $\mathcal{D}_{R}(r, t)$ that occur in ${ }^{p} \underline{H}_{u}{ }^{p} \underline{H}_{t}$. Therefore, the only $p$-canonical basis elements that are indexed by elements in $\sigma_{z}$ and that occur with non-trivial coefficient in (5) are the following:

$$
\sum_{1 \leqslant j \leqslant m-1} a_{j, i}\left(\delta_{z_{j+1} \in \sigma_{z}}^{p} \underline{H}_{z_{j+1}}+\delta_{z_{j-1} \in \sigma_{z}}^{p} \underline{H}_{z_{j-1}}\right)
$$

On the other hand we can rewrite the product as follows:

$$
\begin{aligned}
{ }^{p} \underline{H}_{s}{ }^{p} \underline{H}_{x_{i}}{ }^{p} \underline{H}_{t} & ={ }^{p} \underline{H}_{s}\left(\sum_{u \leqslant x_{i} t} \mu_{x_{i}, t}^{u} \underline{H}_{u}\right) \\
& =\sum_{\substack{w \leqslant x_{i} t \\
s w<w}}\left(v+v^{-1}\right)^{p} \mu_{x_{i}, t}^{w} \underline{H}_{w}+\sum_{\substack{u \leqslant x_{i} t \wedge w \leqslant s u \\
s u>u}}{ }^{p} \mu_{x_{i}, t}^{u}{ }^{p} \mu_{s, u}^{w} \underline{H}_{w}
\end{aligned}
$$

Consider an element $u \leqslant x_{i} t$ with ${ }^{p} \mu_{x_{i}, t}^{u} \neq 0$. It follows that $u$ has $t$ in its right descent set. The version of Lemma 3.4 for left cells shows that ${ }^{p} \underline{H}_{s}{ }^{p} \underline{H}_{u}$ can only contribute $p$-canonical basis elements indexed by elements in $\mathcal{D}_{R}(r, t)$ if $u$ lies in $\mathcal{D}_{R}(r, t)$ itself. (Observe that Corollary 4.8 together with the fact that elements in $\sigma_{x}$ have the same left descent set implies that the sum $\sum_{\substack{w \leqslant x_{i} t \\ s w<}}\left(v+v^{-1}\right)^{p} \mu_{x_{i}, t}^{w} \underline{H}_{w}$ cannot contribute any $p$-canonical basis elements indexed by elements in $\sigma_{z}$.) Using Corollary 4.8 and Corollary 3.13 again, we see that the only $p$-canonical basis elements that are indexed by elements in $\sigma_{z}$ and that occur with non-trivial coefficient in (6) are the following:

$$
\sum_{1 \leqslant j \leqslant m-1}\left(\delta_{x_{i+1} \in \sigma_{x}} a_{j, i+1}+\delta_{x_{i-1} \in \sigma_{x}} a_{j, i-1}\right)^{p} \underline{H}_{z_{j}}
$$

Comparing coefficients in front of ${ }^{p} \underline{H}_{z_{j}}$ we get:

$$
\delta_{z_{j-1} \in \sigma_{z}} a_{j-1, i}+\delta_{z_{j+1} \in \sigma_{z}} a_{j+1, i}=\delta_{x_{i+1} \in \sigma_{x}} a_{j, i+1}+\delta_{x_{i-1} \in \sigma_{x}} a_{j, i-1}
$$

Letting $i$ and $j$ vary, we see that the $a_{j, i}$ 's satisfy precisely the inequalities (i) - (xiii) and (i') (xii') (with equality).

Corollary 4.10. Let $x, y \in \mathcal{D}_{R}(r, t)$ and $s \in S$ such that $s x>x$. Then we have

$$
{ }^{p} \mu_{s, x}^{y}={ }^{p} \mu_{s, x^{*}}^{y^{*}}
$$

where $(-)^{*}$ is the right star-operation with respect to $\{r, t\}$.

Using the $\mathbb{Z}\left[v, v^{-1}\right]$-linear anti-involution $\iota$ on $\mathcal{H}$ we can translate all the results in this section about right strings and right star-operations into results about left strings and left star-operations.

\subsection{Consequences for $p$-Cells}

As we have seen in the last section one needs some assumptions on $p$ for the left and right staroperations to be well-behaved. Therefore, we keep these assumptions throughout this subsection. Fix for the rest of the section two simple reflections $r, t \in S$ with $3 \leqslant m:=m_{r, t}<\infty$. Throughout the section we consider the right star-operation $(-)^{*}$ with respect to $\{r, t\}$.

Under these assumptions, the $p$-cells in any finite Weyl group of rank 2 coincide with the KazhdanLusztig cells. Therefore, Theorem 3.9 implies for $I=\{r, t\} \subseteq S$ : 
Lemma 4.11. Let $\sigma$ be any right $\langle r, t\rangle$-string. Then all elements of $\sigma$ lie in the same right $p$-cell of $W$.

In particular, we have the following result:

Corollary 4.12. For all $x \in \mathcal{D}_{R}(r, t), x$ and $x^{*}$ lie in the same right $p$-cell.

The following important result follows from Corollary 4.10:

Theorem 4.13. For $x, y \in \mathcal{D}_{R}(r, t)$ we have:

$$
x \underset{L}{\stackrel{p}{\leqslant}} y \Leftrightarrow x^{*} \underset{L}{\stackrel{p}{\leqslant}} y^{*}
$$

In particular, if $x$ and $y$ lie the same left $p$-cell, then the same holds for $x^{*}$ and $y^{*}$.

Definition 4.14. For $r, t \in S$ with $r t \neq t r$ and $x \in \mathcal{D}_{R}(r, t)$ we denote by $\sigma_{x}$ the right $\langle r, t\rangle$-string through $x$. Define $\mathfrak{T}_{r, t}(x):=\{x r, x t\} \cap \mathcal{D}_{R}(r, t)$ to be the neighbouring elements of $x$ in $\sigma_{x}$.

View $\mathfrak{T}_{r, t}$ as a map $\sigma_{x} \rightarrow \mathcal{P}\left(\sigma_{x}\right)$ where $\mathcal{P}\left(\sigma_{x}\right)$ denotes the power set of $\sigma_{x}$. We define $\mathfrak{T}_{r, t}^{2}$ : $\sigma_{x} \rightarrow \mathcal{P}\left(\sigma_{x}\right)$ to be the map sending $y \in \sigma_{x}$ to $\bigcup_{z \in \mathfrak{T}_{r, t}(y)} \mathfrak{T}_{r, t}(z)$. For $l \geqslant 2$, the map $\mathfrak{T}_{r, t}^{l}$ is defined inductively in a similar fashion.

Actually, one can characterize precisely the possible left $p$-cell preorder relations among elements in right $\langle r, t\rangle$-strings:

Proposition 4.15. Let $\sigma=\left\{x_{1}<x_{2}<\ldots\right\}$ (resp. $\sigma^{\prime}=\left\{y_{1}<y_{2}<\ldots\right\}$ ) be two right $\langle r, t\rangle$-strings consisting of $m-1$ elements. Up to possibly interchanging the roles of $\sigma$ and $\sigma^{\prime}$, the set of left $p$-cell preorder relations between the elements of these two strings is one of the following:

$$
\begin{aligned}
& \text { no relation: }\{\} \\
& \text { trivial case: }\left\{x_{i} \underset{L}{\stackrel{p}{\leqslant}} y_{i} \mid \forall 1 \leqslant i \leqslant m-1\right\} \\
& \text { permuted case: }\left\{x_{i} \underset{L}{\stackrel{p}{\leftarrow}} y_{\pi(i)} \mid \forall 1 \leqslant i \leqslant m-1\right\} \\
& \text { neighbour case: }\left\{x_{i} \underset{L}{\stackrel{p}{\leqslant}} y \mid \forall 1 \leqslant i \leqslant m-1, y \in \mathfrak{T}_{r, t}^{k}\left(y_{i}\right)\right\} \\
& \underset{\text { neighour case: }}{\text { permuted }}\left\{x_{i} \underset{L}{\stackrel{p}{\leqslant}} y_{\pi(j)} \mid \forall 1 \leqslant i \leqslant m-1, y_{j} \in \mathfrak{T}_{r, t}^{l}\left(y_{i}\right)\right\} \\
& \text { zig-zag case: }\left\{x_{i-1} \underset{L}{\stackrel{p}{\leqslant}} y_{i+1}, x_{i} \underset{L}{\stackrel{p}{\leqslant}} y_{i}, x_{i+1} \underset{L}{\stackrel{p}{\leqslant}} y_{i-1} \mid \forall 2 \leqslant i \leqslant m-2\right\}
\end{aligned}
$$

for $1 \leqslant k \leqslant m-2,1 \leqslant l \leqslant m-4$ where $\pi=(1, m-1)(2, m-2)$ is a permutation of $\{1,2, \ldots, m-1\}$.

Proof. The proof strategy is as follows: By applying Proposition 4.9 to the relation $y \frac{p}{L} x$ via $s \in S$, we get other elementary left $p$-cell relations between the elements in $\sigma_{x}$ and $\sigma_{y}$. The idea is to encode them in a function $f: \sigma_{y} \rightarrow \mathcal{P}\left(\sigma_{x}\right)$ such that

$$
{ }^{p} \mu_{s, y_{i}}^{x_{j}} \neq 0 \Leftrightarrow x_{j} \in f\left(y_{i}\right) .
$$

The claim of the proposition is that there is a normal form of arbitrary finite compositions of such functions. If $g: \sigma_{x} \rightarrow \mathcal{P}\left(\sigma_{z}\right)$ is another such function, their composition $g \circ f: \sigma_{y} \rightarrow \mathcal{P}\left(\sigma_{z}\right)$ sends $y_{i}$ to $\bigcup_{x_{j} \in f\left(y_{i}\right)} g\left(x_{j}\right)$. This is simply the composition of multi-valued functions.

In order to simplify notation, we will identify the $j$-th element in a right $\langle r, t\rangle$-string with its position $j$. This allows us to view any such $f$ as a map $\{1,2, \ldots, m-1\} \rightarrow \mathcal{P}(\{1,2, \ldots, m-1\})$. The composition of such functions is to be understood in a similar fashion. One needs to keep track 
of the start and end string of the whole composition in order to retranslate the function into the set of left $p$-cell relations. Using Corollary 4.10 we see that such a map is already fully determined by the images of $1,2, \ldots,\left\lceil\frac{m-1}{2}\right\rceil$.

We will prove the statement for $m=6$ and leave the other cases to the reader. Apart from the identity Id $: i \mapsto\{i\}$ consider the following maps where we identify by slight abuse of notation the permutation $\pi$ with the induced map $\{1,2, \ldots, 5\} \rightarrow \mathcal{P}(\{1,2, \ldots, 5\})$ :

$$
\begin{array}{rlrl}
1 & \mapsto\{3\} & 1 & \mapsto\{2\} \\
a: 2 & \mapsto\{2,4\} & b:\{1,3\} \\
3 & \mapsto\{1,3,5\} & & \mapsto \mapsto\{2,4\} \\
1 & \mapsto\{4\} & \mapsto: 2 & \mapsto\{4\} \\
c: 2 & \mapsto\{3,5\} & 3 & \mapsto\{3\}
\end{array}
$$

Using Proposition 4.9 it is easy to check that any elementary left $p$-cell relation implies relations encoded by one of the functions above, for example $3 \underset{L}{\stackrel{p}{\rightarrow}} 3$ implies relations encoded by either Id, $a$ or $\pi$. Analyzing the relations among compositions of these functions, one has:

$$
\begin{aligned}
a \circ \pi & =\pi \circ a=a \\
b \circ \pi & =\pi \circ b=c \\
b \circ c & =c \circ b \\
\pi^{2} & =\mathrm{Id} \\
a \circ b & =b \circ a=b^{3} \\
a^{k}=a^{2} & =b^{4}=b^{2 k}=b^{2 k} \circ \pi \text { for } k \geqslant 2 \\
b^{3} & =b^{2 k+1}=b^{2 k+1} \circ \pi \text { for } k \geqslant 1
\end{aligned}
$$

Using these relations, we see that any finite composition of these maps can be reduced to one of the following compositions

$$
\mathrm{Id}, \pi, b^{k} \text { for } 1 \leqslant k \leqslant 4, b^{l} \circ \pi \text { for } 1 \leqslant l \leqslant 2, a .
$$

These correspond precisely to the cases with at least one relation stated in the proposition.

Remark 4.16. It should be noted that for $m<6$ some of these cases coincide. For example for $m=3$, the permutation $\pi$ is trivial and there are only three distinct cases: The permuted case coincides with the trivial case. Moreover, the zig-zag case does not contain any relations and the permuted neighbour case does not exist. For $m=4$, there are four distinct cases: The zig-zag case reduces to the permuted case and the permuted neighbour case does not exist.

The normal forms given in the proof of Proposition 4.15 allow us to deduce the following equivalences, which show how rigid the combinatorics in this situation are. The reader should compare these equivalences with Proposition 4.9 which only deals with the generating relations for the $p$-cell preorder. It is a generalization of [Shi94, Proposition 4.6 and Remark 4.7]:

Corollary 4.17. Let $\sigma=\left\{x_{1}<x_{2}<\ldots\right\}$ (resp. $\sigma^{\prime}=\left\{y_{1}<y_{2}<\ldots\right\}$ ) be two right $\langle r, t\rangle$-strings consisting of $m-1$ elements. In addition to $x \underset{L}{\stackrel{p}{\leqslant}} y \Leftrightarrow x^{*} \underset{L}{\stackrel{p}{\leqslant}} y^{*}$ for $x \in \sigma$ and $y \in \sigma^{\prime}$ we get the 
following equivalences:

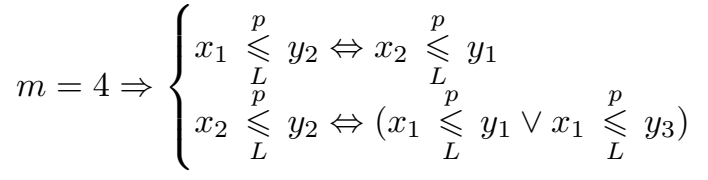

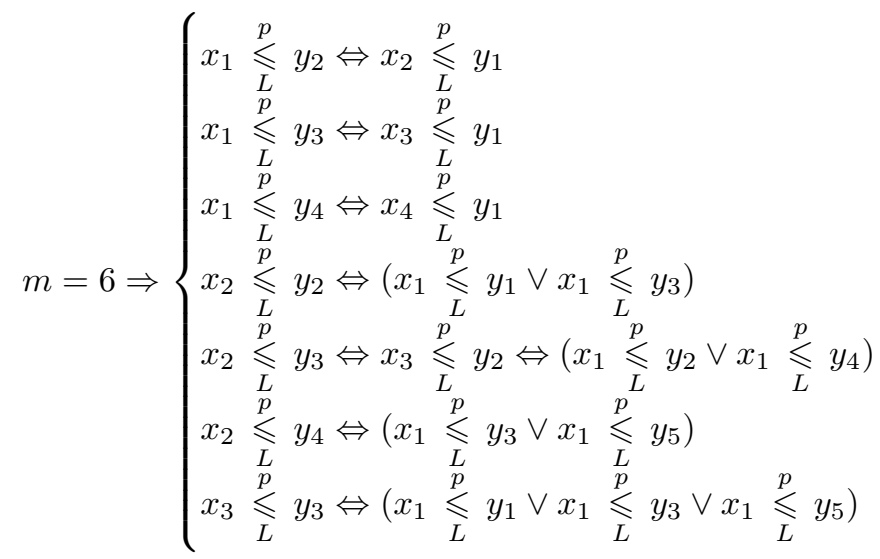

We can now generalize [Lus85, Proposition 10.7] as follows:

Proposition 4.18. Let $r, t \in S$ and $\Gamma$ be a union of left $p$-cells such that $\Gamma \subseteq \mathcal{D}_{R}(r, t)$. Then the following holds:

(i) $\widetilde{\Gamma}:=\left(\bigcup_{w \in \Gamma} \sigma_{w}\right) \backslash \Gamma$ is a union of left p-cells.

(ii) If $\Gamma$ is a left $p$-cell, then $\widetilde{\Gamma}$ is a union of at most $m-2$ left $p$-cells.

(iii) If $\Gamma$ is a left $p$-cell, then $\Gamma^{*}:=\left\{w^{*} \mid w \in \Gamma\right\}$ is a left $p$-cell as well.

Proof. For two left $p$-cells $\Gamma_{1}$ and $\Gamma_{2}$ contained in $\mathcal{D}_{R}(r, t)$ we have:

$$
\begin{aligned}
\left(\widetilde{\Gamma_{1} \cup \Gamma_{2}}\right) & =\left(\bigcup_{w \in \Gamma_{1} \cup \Gamma_{2}} \sigma_{w}\right) \backslash\left(\Gamma_{1} \cup \Gamma_{2}\right) \\
& =\widetilde{\Gamma_{1}} \backslash \Gamma_{2} \cup \widetilde{\Gamma_{2}} \backslash \Gamma_{1}
\end{aligned}
$$

Therefore, we may assume without loss of generality that $\Gamma$ is a left $p$-cell. It is enough to prove that $\bigcup_{w \in \Gamma} \sigma_{w}$ is a union of left $p$-cells.

Let $x \in \bigcup_{w \in \Gamma} \sigma_{w}$ and $y \in W$ such that $x \underset{L}{\stackrel{p}{\sim}} y$. Then there exists a sequence

$$
P: x=x_{0} \underset{L}{\stackrel{p}{\rightarrow}} x_{1} \underset{L}{\stackrel{p}{\rightarrow}} \cdots \underset{L}{\rightarrow} x_{k}=y \underset{L}{\stackrel{p}{\rightarrow}} x_{k+1} \underset{L}{\stackrel{p}{\rightarrow}} \cdots \frac{p}{\rightarrow} x_{l}=x
$$

of elements in $\mathcal{D}_{R}(r, t)$ (by Lemma 3.4) that all lie in the same left $p$-cell as $x$ and $y$. Consider the right $\langle r, t\rangle$-strings of all the elements in the sequence and note that $\sigma_{x}$ contains an element $\bar{x} \in \Gamma$. Since each $\langle r, t\rangle$-string is contained in a right $p$-cell (see Lemma 4.11), the elements have the same left descent set. Thus for $0 \leqslant i<l$ all the elements $z \in \sigma_{x_{i}}$ satisfy $s_{i} z>z$ where $s_{i}$ is the simple reflection used to get from $x_{i}$ to $x_{i+1}$. Express $\underline{H}_{s_{i}}{ }^{p} \underline{H}_{z}$ in the $p$-canonical basis and remember the elements in $\sigma_{x_{i+1}}$ indexing $p$-canonical basis elements with non-trivial coefficients. This gives the elementary left $p$-cell relations between $\sigma_{x_{i}}$ and $\sigma_{x_{i+1}}$ induced by $s_{i}$. By combining the various elementary left $p$-cell relations between $\sigma_{x_{i}}$ and $\sigma_{x_{i+1}}$ induced by $s_{i}$ along the sequence $P$, we obtain for any $z \in \sigma_{x}$ 
a possibly empty set $f_{P}(z)$ of elements in $\sigma_{x}$ such that $z \underset{L}{p} z^{\prime}$ if and only if $z^{\prime} \in f_{P}(z)$. The proof of Proposition 4.15 shows that for $n \gg 1$ the image of $f_{P}^{2 n}$ stabilizes in the sense that $z \in f_{P}^{2 n}(z)$. The element $\bar{x}$ shows that there is an element in $\sigma_{y} \cap \Gamma$ which implies $y \in \bigcup_{w \in \Gamma} \sigma_{w}$ and finishes the proof of (i).

We claim that any left $p$-cell $\Gamma^{\prime}$ in $\bigcup_{w \in \Gamma} \sigma_{w}$ intersects $\sigma_{x}$ non-trivially. Let $y \in \Gamma^{\prime}$ and $\bar{y} \in \sigma_{y} \cap \Gamma$. Use a sequence $P$ as above relating $\bar{x}$ and $\bar{y}$ to construct $f_{P}: \sigma_{x} \rightarrow \mathcal{P}\left(\sigma_{x}\right)$. Proposition 4.15 shows that there exist $x^{\prime} \in \sigma_{x}$ such that $x^{\prime} \stackrel{p}{L}_{y}$. Using the stabilization of $f_{P}$ as above, we get $x^{\prime} \in f_{P}^{2 n}\left(x^{\prime}\right)$ for $n \gg 1$ and in particular $x^{\prime}$ lies in $\Gamma^{\prime} \cap \sigma_{x}$. This finishes the proof of the claim and shows that $\widetilde{\Gamma}$ is a union of at most $m-2$ left $p$-cells giving (ii).

(iii) is an immediate consequence of Theorem 4.13 .

Remark 4.19. Analyzing the situation more carefully allows us to say more about the number of left $p$-cells in $\widetilde{\Gamma}$. Let $x, y \in \Gamma$. Consider $f_{P}^{n}$ constructed as in the last proof for a sequence $P$ relating $x$ and $y$. The proof of Proposition 4.15 shows that $f_{P}^{2 n}$ for $n \gg 1$ stabilizes to one of the following maps:

$$
\begin{aligned}
f_{\text {triv }}: \sigma_{x} \longrightarrow \mathcal{P}\left(\sigma_{x}\right) \quad \text { or } \quad f_{\text {nontriv }}: \sigma_{x} & \longrightarrow \mathcal{P}\left(\sigma_{x}\right) \\
x_{i} \quad \longmapsto\left\{x_{i}\right\} \quad & \longmapsto\left\{x_{2 k-1} \mid 1 \leqslant k \leqslant\left\lfloor\frac{m}{2}\right\rfloor\right\} \\
x_{2 l} & \longmapsto\left\{x_{2 k} \mid 1 \leqslant k \leqslant\left\lfloor\frac{m-2}{2}\right\rfloor\right\}
\end{aligned}
$$

Finally, let $P$ vary over all potential sequences and $y$ vary over the elements in $\Gamma$. If there exists an element $y \in \Gamma$ and a sequence $P$ relating $x$ and $y$ such that $f_{P}^{n}$ stabilizes to $f_{\text {nontriv }}$, then $\widetilde{\Gamma}$ is a left $p$-cell (note that $\widetilde{\Gamma}$ is always non-empty as all elements in $\Gamma$ have the same right descent set and this is not the case for all elements in $\sigma_{x}$ ).

Another situation, in which we can say more about the number of $p$-cells in $\widetilde{\Gamma}$ is the following: If $m \geqslant 4$ and there exists a string $\sigma$ such that $\sigma \cap \Gamma$ contains only one odd-numbered element of $\sigma$, then $\widetilde{\Gamma}$ decomposes into at least 2 left $p$-cells for descent set reasons. Therefore, under these assumptions $\widetilde{\Gamma}$ contains precisely two right $p$-cells if $m=4$.

The definition of a $W$-graph from [KL79, §1] describes a based representation of the Hecke algebra. A typical example is given by a Kazhdan-Lusztig cell module equipped with the Kazhdan-Lusztig basis, for which the $W$-graph describes the action of the generating set $\left\{H_{s} \mid s \in S\right\}$ of the Hecke algebra. In order to allow $W$-graphs to also describe $p$-cell modules, we need to slightly generalize the original definition, in which we use the generating set $\left\{\underline{H}_{s} \mid s \in S\right\}$ of the Hecke algebra instead:

Definition 4.20. A coloured $W$-graph is a directed graph with vertices $\mathcal{V}$ and edges $\mathcal{E}$ together with the following decorations:

- for each vertex $x \in \mathcal{V}$ a subset $I_{x}$ of $S$,

- for each edge $(x, y) \in \mathcal{E}$ a family of Laurent polynomials

$$
\left\{\mu_{s, x}^{y} \mid s \in I_{y} \backslash I_{x}\right\} \subset \mathbb{Z}\left[v, v^{-1}\right]
$$

subject to the conditions below. Let $V$ be the free $\mathbb{Z}\left[v, v^{-1}\right]$-module with basis $\mathcal{V}$. For $s \in S$ define a $\mathbb{Z}\left[v, v^{-1}\right]$-linear endomorphism of $V$ as follows

$$
\tau_{s}(x)= \begin{cases}\left(v+v^{-1}\right) x & \text { if } s \in I_{x} \\ \sum_{\substack{(x, y) \in \mathcal{E} \\ s \in I_{y}}} \mu_{s, x}^{y} y & \text { otherwise }\end{cases}
$$


where the sum is finite due to the second condition below. Then a coloured $W$-graph is required to satisfy:

(i) the morphism $\mathcal{H} \rightarrow \operatorname{End}(V), \underline{H}_{s} \mapsto \tau_{s}$ extends to a morphism of $\mathbb{Z}\left[v, v^{-1}\right]$-algebras,

(ii) for each pair $(x, s) \in \mathcal{V} \times S$ there are only finitely many edges $(x, y) \in \mathcal{E}$ with $\mu_{s, x}^{y} \neq 0$.

It is immediate that we can associate to each left $p$-cell $C$ a coloured $W$-graph $\Gamma_{C}$ as defined above with $C$ as vertex set. For each $x \in C$ we set $I_{x}:=\mathcal{L}(x)$ and use edges to encode the structure coefficients for the $p$-canonical basis ${ }^{p} \mu_{s, x}^{y}$ for $x, y \in C$. Observe that right star operations do not modify the left descent set. This combined with Proposition 4.18 (iii) and Corollary 4.10 implies the following result:

Lemma 4.21. Let $C$ be a left $p$-cell contained in $\mathcal{D}_{R}(r, t)$. Then the left $p$-cell module associated to $C$ is isomorphic to the left p-cell module associated to $C^{*}$ where $*$ is the right star-operation associated to $r$ and $t$. In particular, the coloured $W$-graphs $\Gamma_{C}$ and $\Gamma_{C^{*}}$ are isomorphic as decorated, directed graphs.

\subsection{Vogan's Generalized $\tau$-Invariant}

Vogan defined in [Vog79, Definition 3.10] an invariant of Kazhdan-Lusztig left cells in the setting of primitive ideals for semi-simple Lie algebras. This became known as Vogan's generalized $\tau$-invariant and was generalized in [BG15, Definition 5.1] to arbitrary Coxeter groups. (Observe that only pairs of simple reflections $\{r, t\} \in W$ with $m_{r, t} \in\{3,4\}$ matter in the following definition.)

Definition 4.22. Since $\mathfrak{T}_{r, t}(x)$ for $r, t \in S$ consists of one or two elements, we use the following convention: We consider $\mathfrak{T}_{r, t}(x)$ as a multiset with two identical elements in the following if $\{x r, x t\} \cap$ $\mathcal{D}_{R}(r, t)$ is of cardinality 1 .

We define a sequence of equivalence relations $\approx_{n}$ for $n \in \mathbb{N}$ as follows. For $x, y \in W$ we write:

$$
\begin{aligned}
& x \approx_{0} y \text { if } \mathcal{R}(x)=\mathcal{R}(y), \\
& x \approx_{n+1} y \text { if } x \approx_{n} y \text { and for any pair } r, t \in S \text { such that } m_{r, t} \in\{3,4\} \text { and } \\
& x, y \in \mathcal{D}_{R}(r, t) \text { with } \mathfrak{T}_{r, t}(x)=\left\{x_{1}, x_{2}\right\} \text { and } \mathfrak{T}_{r, t}(y)=\left\{y_{1}, y_{2}\right\} \\
& \quad \text { we have: } x_{1} \approx_{n} y_{1}, x_{2} \approx_{n} y_{2} \text { or } x_{1} \approx_{n} y_{2}, x_{2} \approx_{n} y_{1}
\end{aligned}
$$

We say that $x$ and $y$ have the same generalized $\tau$-invariant if $x \approx_{n} y$ holds for all $n \geqslant 0$. We call the set

$$
\left\{w \in W \mid x \approx_{n} w \text { for all } n \geqslant 0\right\}
$$

the $\tau$-equivalence class of $x$.

Remark 4.23. Observe that the last definition admits an obvious generalization allowing the case $m_{r, t}=6$. For our current applications, we do not need this generality. Thus we exclude this case just like Vogan did in the original definition.

The following result shows that left $p$-cells give a refinement of the $\tau$-equivalence classes. It is a generalization of [BG15, Theorem 5.2]:

Theorem 4.24. Assume $p>2$ if there exist $s \neq t \in S$ with $m_{s, t}=4$. Let $\Gamma$ be a left p-cell. Then all elements in $\Gamma$ have the same generalized $\tau$-invariant. In particular, any $\tau$-equivalence class decomposes into left $p$-cells.

Proof. The proof of [BG15, Theorem 5.2] works after replacing all Kazhdan-Lusztig related constructions by their $p$-canonical analogues with the following modifications: First add a zeroth case in which there exists a right $\langle s, t\rangle$-string $\sigma$ such that $\Gamma \cap \sigma=\left\{x^{\prime} s, x^{\prime} s t s\right\}$ for some minimal element $x^{\prime}$ in its right $\langle s, t\rangle$-coset. In this case, $\widetilde{\Gamma}$ consists of a single left $p$-cell, which allows us to conclude. Finally in the second case, use the left $p$-cell preorder relations for the roles of $y$ and $w$ swapped instead of appealing to [Lus85, Corollary 6.3]. 
Corollary 4.25. Assume $p>2$ if there exist $s \neq t \in S$ with $m_{s, t}=4$. Assume that two elements in $W$ have the same generalized $\tau$-invariant if and only if they belong to the same Kazhdan-Lusztig left cell (i.e. the generalized $\tau$-invariant gives a complete invariant of Kazhdan-Lusztig left cells). Then Kazhdan-Lusztig left cells decompose into left p-cells.

In [Vog79, Theorem 6.5], Vogan shows that the generalized $\tau$-invariant gives a complete invariant in finite type $A$. The same holds in finite types $B / C$ (see [Gar93, Theorem 3.5.9] based on [Gar92; Gar93, Definitions 2.1.3. - 2.1.7., 3.2.1. and 3.4.1.]) and finite type $E_{6}$ (see [Ton95]). Therefore, we have:

Corollary 4.26. The Kazhdan-Lusztig left cells in finite type A decompose into left p-cells. The same holds in finite types $B$ and $C$ for $p>2$ and in finite type $E_{6}$.

Even though we currently can only calculate the full $p$-canonical basis in types $B_{n}$ and $C_{n}$ for $n \leqslant 5$ and in these groups only 2-torsion occurs, the last result is in particular of interest due to [Wil17b; Wil17a]. In these papers Williamson shows that there is torsion in the local integral intersection cohomology of Schubert varieties in the flag variety of the general linear group that grows exponentially in the rank. This implies that the $p$-canonical basis does not coincide with the Kazhdan-Lusztig basis for arbitrarily large primes in type $A_{n}$ (and that the primes for which this occurs grow exponentially in the rank). Since type $A_{n}$ embeds into type $B_{n+1}$ and $C_{n+1}$ this gives many interesting examples for which the $p$-canonical basis and the Kazhdan-Lusztig basis differ for large primes $p$.

In [Vog79, Remark following Proposition 4.4] Vogan mentions that the generalized $\tau$-invariant is not a complete invariant in type $F_{4}$. Similarly in type $D_{n}$ for $n \geqslant 6$ (see the introduction of [Gar90]).

As proposed by [Vog80, §4] and [BG15, Remark 5.3] we could have defined an invariant as follows:

Definition 4.27. As in Definition 4.27 we define a sequence of equivalence relations $\approx_{n}^{\prime}$ for $n \in \mathbb{N}$ as follows. For $x, y \in W$ we write:

$$
\begin{aligned}
& x \approx_{0}^{\prime} y \text { if } \mathcal{R}(x)=\mathcal{R}(y), \\
& x \approx_{n+1}^{\prime} y \text { if } x \approx_{n}^{\prime} y \text { and for any pair } r, t \in S \text { such that } \infty>m_{r, t} \geqslant 3 \text { and } \\
& x, y \in \mathcal{D}_{R}(r, t) \text { we have: } x^{*} \approx_{n}^{\prime} y^{*}
\end{aligned}
$$

where $(-)^{*}$ is the right star-operation with respect to $\{r, t\}$. As above we say that $x$ and $y$ have the same generalized $\widetilde{\tau}$-invariant if $x \approx_{n}^{\prime} y$ holds for all $n \geqslant 0$. We call the set

$$
\left\{w \in W \mid x \approx_{n}^{\prime} w \text { for all } n \geqslant 0\right\}
$$

the $\widetilde{\tau}$-equivalence class of $x$.

In this case, Theorem 4.13 immediately implies that the partition of $W$ into left $p$-cells gives a refinement of the $\widetilde{\tau}$-equivalence classes:

Corollary 4.28. Assume $p>2$ (resp. $p>3$ ) if there exist $s \neq t \in S$ with $m_{s, t}=4$ (resp. $\left.m_{s, t}=6\right)$. Let $\Gamma$ be a left $p$-cell. Then all elements in $\Gamma$ have the same generalized $\widetilde{\tau}$-invariant. In particular, any $\widetilde{\tau}$-equivalence class decomposes into left $p$-cells.

In [GH15, Conjecture 6.9] Geck and Halls propose a slight variation of Vogan's original conjecture (see [Vog79, Conjecture 3.11]) which goes as follows:

\section{Conjecture 4.29.}

For any finite Coxeter group $W_{f}$ two elements $x, y \in W_{f}$ belong to the same Kazhdan-Lusztig left cell if and only if $x$ and $y$ lie in the same Kazhdan-Lusztig two-sided cell and in the same $\widetilde{\tau}$-equivalence class. 
They verified their conjecture in all types $B C_{n}$ and $D_{n}$ for $n \leqslant 9$ and in all exceptional types. Moreover, they mention that in type $F_{4}$ the Kazhdan-Lusztig left cells are precisely the $\widetilde{\tau}$-equivalence classes. From this, we deduce that for $p \geqslant 3$ the Kazhdan-Lusztig left cells in type $F_{4}$ decompose into left $p$-cells. Our explicit computer calculations show that the $p$-canonical basis and the KazhdanLusztig basis in type $F_{4}$ only differ for $p \in\{2,3\}$ and for $p=2$ the Kazhdan-Lusztig left cells do not decompose into left $p$-cells.

\section{$4.4 p$-Cells in Type $A$}

Kazhdan-Lusztig cells in type $A$ can be characterized using the Robinson-Schensted correspondence. This result is usually attributed to $[\mathrm{KL} 79, \S 4]$ even though the result is not stated in this form and depends on results of Joseph and Vogan in the setting of primitive ideals. The first combinatorial proof is due to [GM88] and [Ari00]. In this section we transfer almost verbatim Ariki's proof to the modular setting. Since we feel that the proof is not as well documented as it should be, we decided to give the proof here.

Throughout this section we assume that we used the Cartan matrix in finite type $A_{n-1}$ as input. In this case $(W, S)$ can be identified with $\left(S_{n},\left\{s_{1}, \ldots, s_{n-1}\right\}\right)$ the symmetric group together with the set of simple transpositions. Letting $S_{n}$ act on $\{1,2, \ldots, n\}$ on the left, we can write any element $w \in S_{n}$ uniquely as $w=w(1) w(2) \ldots w(n)$ which we call string notation.

Recall the definition of the elementary Knuth transformation $K_{i}$ for $1<i<n$ : Let $x=$ $x_{1} x_{2} \ldots x_{n}, y=y_{1} y_{2} \ldots y_{n} \in S_{n}$ be two elements of the symmetric group in string notation. Write $x \underset{K_{i}}{\approx} y$ if $x$ and $y$ differ only on the substrings $x_{i-1} x_{i} x_{i+1}$ and $y_{i-1} y_{i} y_{i+1}$ and these substrings are related to each other in either of the following two ways

$$
b c a \leftrightarrow b a c \text { or } c a b \leftrightarrow a c b
$$

where $a<b<c$. We say that $x$ and $y$ are Knuth equivalent if they are related by a sequence of elementary Knuth transformations $K_{i}$ for various $i$. The following result follows immediately from the definitions:

Lemma 4.30. $\mathcal{D}_{R}\left(s_{i-1}, s_{i}\right)$ is the subset of elements in $S_{n}$ to which $K_{i}$ can be applied. Moreover, $w \underset{K_{i}}{\approx} w^{*}$ for all $w \in \mathcal{D}_{R}\left(s_{i-1}, s_{i}\right)$ where $(-)^{*}$ is the right star-operation defined with respect to $\left\{s_{i-1}, s_{i}\right\}$.

The Robinson-Schensted correspondence (see [BB05, §A.3.3] or [Ful97, §4.1]) gives a bijection between the symmetric group $S_{n}$ and pairs of standard tableaux of the same shape with $n$ boxes. The row-bumping algorithm gives a way to explicitly calculate the image $(P(w), Q(w))$ of $w \in S_{n}$ under the Robinson-Schensted correspondence. We will need the following important classical result about the Robinson-Schensted correspondence (see [Ful97, §4.1, Corollary to Symmetry Theorem]):

Theorem 4.31 (Symmetry Theorem for $S_{n}$ ).

If $w \in S_{n}$ corresponds to $(P(w), Q(w))$, then $w^{-1}$ corresponds to $(Q(w), P(w))$ under the RobinsonSchensted correspondence.

Moreover, we need the following result by Knuth (see [Knu70, Theorem 6]):

Theorem 4.32. Let $x, y \in S_{n}$. Then $x$ and $y$ are Knuth equivalent if and only if $P(x)=P(y)$.

The goal is to prove the following theorem which is known for Kazhdan-Lusztig cells (see [Wil03, Theorem 5.4.1 and Corollary 5.4.2] or [BB05, Chapter 6, Exercise 11]):

Theorem 4.33. For $x, y \in S_{n}$ we have:

$$
x \underset{L}{\stackrel{p}{\sim}} y \Leftrightarrow Q(x)=Q(y)
$$




$$
\begin{aligned}
& x \underset{R}{\stackrel{p}{\sim}} y \Leftrightarrow P(x)=P(y) \\
& x \underset{L R}{\stackrel{p}{\sim}} y \Leftrightarrow Q(x) \text { and } Q(y) \text { have the same shape }
\end{aligned}
$$

In particular, Kazhdan-Lusztig cells and p-cells of $S_{n}$ coincide.

Remark 4.34. To prove the characterization of the left $p$-cell cells we only need the following ingredients: For the direction $\Leftarrow$ we need Lemma 3.6 and Corollary 4.12. The other direction is more involved and relies on the version of Lemma 3.4 for left cells and crucially on Theorem 4.13.

Proof. We will first deal with the statement about left $p$-cells:

$\Leftarrow$ By Theorem 4.31 we have $P\left(x^{-1}\right)=Q(x)=Q(y)=P\left(y^{-1}\right)$ which implies by Theorem 4.32 that $x^{-1}$ and $y^{-1}$ are related by a sequence of elementary Knuth transformations (i.e. right start operations with respect to different subsets of $S$ consisting of two neighbouring simple reflections). Successive applications of Corollary 4.12 show that $x^{-1} \underset{R}{\stackrel{p}{\sim}} y^{-1}$. This is equivalent to $x \underset{L}{\stackrel{p}{\sim}} y$ by Lemma 3.6.

$\Rightarrow$ Denote the shape of $Q(x)$ (resp. $Q(y))$ by $\pi_{x}$ (resp. $\left.\pi_{y}\right)$ and let $P_{x}$ (resp. $P_{y}$ ) be the column superstandard tableau (see [BB05, §A3.5] for the definition) of shape $\pi_{x}$ (resp. $\pi_{y}$ ). The RobinsonSchensted correspondence gives elements $\hat{x}, \hat{y} \in S_{n}$ with $P$ and $Q$-symbols $\left(P_{x}, Q(x)\right)$ and $\left(P_{y}, Q(y)\right)$ respectively. The implication we proved above gives $x \underset{L}{\stackrel{p}{\sim}} \hat{x}$ and $y \underset{L}{\stackrel{p}{\sim}} \hat{y}$ which implies by our assumption $\hat{x} \underset{L}{\stackrel{p}{\sim}} \hat{y}$. In order to show $Q(x)=Q(y)$ consider the elements $x^{\prime}, y^{\prime \prime} \in S_{n}$ corresponding to $\left(P_{x}, P_{x}\right)$ and $\left(P_{y}, P_{y}\right)$ respectively (under the Robinson-Schensted correspondence). Theorem 4.32 implies that the elements $\hat{x}$ and $x^{\prime}$ as well as $\hat{y}$ and $y^{\prime \prime}$ are related by sequences of Knuth moves:

$$
\begin{aligned}
x^{\prime} & =K_{i_{r}} \circ \cdots \circ K_{i_{1}}(\hat{x}) \\
y^{\prime \prime} & =K_{j_{s}} \circ \cdots \circ K_{j_{1}}(\hat{y})
\end{aligned}
$$

Lemma 3.4 for the left $p$-cell preorder shows that the elements $\hat{x}$ and $\hat{y}$ have the same right descent set, so the same right star-operations or Knuth moves (see Lemma 4.30) can be applied to both elements. By Theorem 4.13 we have $K_{i_{1}}(\hat{x}) \underset{L}{\stackrel{p}{\sim}} K_{i_{1}}(\hat{y})$ and $K_{j_{1}}(\hat{x}) \underset{L}{\stackrel{p}{\sim}} K_{j_{1}}(\hat{y})$. Therefore, we can repeat the argument to see that the following elements are well-defined:

$$
\begin{aligned}
x^{\prime \prime} & =K_{j_{s}} \circ \cdots \circ K_{j_{1}}(\hat{x}) \\
y^{\prime} & =K_{i_{r}} \circ \cdots \circ K_{i_{1}}(\hat{y})
\end{aligned}
$$

Moreover, we have $x^{\prime} \underset{L}{\stackrel{p}{\sim}} y^{\prime}$ as well as $x^{\prime \prime} \underset{L}{\stackrel{p}{\sim}} y^{\prime \prime}$ and thus $\mathcal{R}\left(x^{\prime}\right)=\mathcal{R}\left(y^{\prime}\right)$ and $\mathcal{R}\left(x^{\prime \prime}\right)=\mathcal{R}\left(y^{\prime \prime}\right)$. Using Theorem 4.32 we see that $P\left(x^{\prime \prime}\right)=P(\hat{x})=P_{x}$ and $P\left(y^{\prime}\right)=P(\hat{y})=P_{y}$.

Denote the column lengths of $\pi_{x}$ (resp. $\pi_{y}$ ) by $l_{1}, l_{2}, \ldots$ (resp. $k_{1}, k_{2}, \ldots$ ). It follows from the row-bumping algorithm that $x^{\prime}$ is the longest element in the parabolic subgroup $S_{l_{1}} \times S_{l_{2}} \times \ldots$ of $S_{n}$, i.e. in string notation the element

$$
l_{1}, l_{1}-1, \ldots, 1, l_{1}+l_{2}, l_{1}+l_{2}-1, \ldots, l_{1}+1, \ldots
$$

From $\mathcal{R}\left(x^{\prime}\right)=\mathcal{R}\left(y^{\prime}\right)$ and the characterization of right descent sets for elements in $S_{n}$ in terms of inversions, we deduce that in the string notation for $y^{\prime}$ the first $l_{1}$ letters are decreasing as well as the next $l_{2}$ letters and so on. Similarly, we may use that $y^{\prime \prime}$ is the longest element in the parabolic subgroup $S_{k_{1}} \times S_{k_{2}} \times \ldots$ of $S_{n}$ and $\mathcal{R}\left(x^{\prime \prime}\right)=\mathcal{R}\left(y^{\prime \prime}\right)$ to deduce that in the string notation of $x^{\prime \prime}$ the first $k_{1}$ letters are decreasing, the next $k_{2}$ letters are in decreasing order, etc. 
Applying the row-bumping algorithm to $y^{\prime}$ to calculate $P\left(y^{\prime}\right)=P_{y}$, we obtain the inequality $k_{1} \geqslant l_{1}$. Using $x^{\prime \prime}$ instead, we get the opposite inequality giving $l_{1}=k_{1}$. Moreover, this shows that when inserting the next $l_{2}$ letters of $y^{\prime}$, no row bumping occurs in the first column (otherwise we would have $k_{1}>l_{1}$ ) and thus we have $k_{2} \geqslant l_{2}$. Again, we may use $x^{\prime \prime}$ to get the opposite inequality and to show $k_{2}=l_{2}$. Repeating the argument, we get $\pi_{x}=\pi_{y}$ and thus $Q\left(y^{\prime}\right)=P_{y}=P_{x}=Q\left(x^{\prime \prime}\right)$ (by the definition of the column superstandard tableau and the fact that $Q(-)$ encodes the order in which boxes are added in the course of the row-bumping algorithm). This shows $y^{\prime}=x^{\prime}=y^{\prime \prime}=x^{\prime \prime}$ as well as $\hat{x}=\hat{y}$ by unravelling the sequences of Knuth moves. Finally, the Robinson-Schensted correspondence gives $Q(x)=Q(y)$ and finishes the proof of the characterization of left $p$-cells in terms of $Q$-symbols.

Using Theorem 4.31 and Lemma 3.6 we obtain the version for right $p$-cells. Finally, we prove the statement about two-sided $p$-cells:

$\Leftarrow$ Theorem 4.32 shows that any two elements of $S_{n}$ with the same $P$-symbol can be related by a sequence of elementary Knuth transformations. Dually, any two elements with the same $Q$-symbol are linked by a sequence of elementary dual Knuth transformations, which we did not introduce, but which correspond to left star-operations. Given an element $x \in S_{n}$ we can thus transform its $P$ and $Q$ symbols using Knuth transformations and their duals into any pair of given standard tableaux of the same shape. Denote by $\pi$ the shape of $Q(x)$ and let $P_{\pi}$ be the column superstandard tableau of shape $\pi$. The statement about left and right $p$-cells shows that $x$ lies in the same two-sided $p$-cell as the element $w_{\pi}$ corresponding to $\left(P_{\pi}, P_{\pi}\right)$ under the Robinson-Schensted correspondence. From this, the reader easily deduces the direction $\Leftarrow$.

$\Rightarrow$ Given an element $x \in S_{n}$, denote by $\pi$ the shape of $Q(x)$. Let $w_{\pi}$ be as defined above. We claim that $y \underset{L R}{\stackrel{p}{\leqslant}} x$ implies $y \underset{L R}{\stackrel{0}{\leqslant}} x$.

Note that $w_{\pi}$ is the longest element in a standard parabolic subgroup of $S_{n}$. Thus we have

$$
{ }^{p} \underline{H}_{w_{\pi}}=\underline{H}_{w_{\pi}} .
$$

As $Q\left(w_{\pi}\right)$ and $Q(x)$ have the same shape, the direction $\Leftarrow \operatorname{implies} w_{\pi} \underset{L R}{\stackrel{p}{\sim}} x$ and $w_{\pi} \underset{L R}{\stackrel{0}{\sim}} x$. The relation $x \underset{L R}{\stackrel{p}{\lessgtr}} w_{\pi}$ together with $(9)$ gives us for all $z \leqslant x$ :

$$
{ }^{p} m_{z, x} \neq 0 \Rightarrow z \underset{L R}{\stackrel{0}{\lessgtr}} w_{\pi}
$$

Therefore, any element $y \underset{L R}{\stackrel{p}{\leqslant}} x$ satisfies $y \underset{L R}{\stackrel{0}{\leqslant}} x$.

Finally, this finishes the proof of the direction $\Rightarrow$ by using the characterization of the Kazhdan-Lusztig two-sided cells in terms of the shape of the $Q$-symbols.

Remark 4.35. Even though Kazhdan-Lusztig left (resp. right) cells coincide with left (resp. right) $p$-cells, it is not known whether the corresponding preorders coincide as well. Leonardo Patimo has a short proof of the fact that the dominance order on partitions is generated by the weak Bruhat order. This implies that the Kazhdan-Lusztig two-sided cell preorder coincides with the two-sided $p$-cell preorder.

Theorem 4.31 implies that the involutions in $S_{n}$ are precisely those elements $w$ that satisfy $P(w)=Q(w)$. This is the only missing observation for the next result: 
Corollary 4.36. Each left p-cell contains a unique involution. Each two-sided p-cell contains the longest element in a standard parabolic subgroup. Let $C_{L}$ (resp. $C_{R}$ ) be a left (resp. right) p-cell. Then we have:

$$
\left|C_{L} \cap C_{R}\right|= \begin{cases}1 & \text { if } C_{L} \text { and } C_{R} \text { lie in the same two-sided } p \text {-cell, } \\ 0 & \text { otherwise. }\end{cases}
$$

Let $\pi$ be a permutation of $n$. Recall Frame, Robinson and Thrall's hook length formula for the number of standard tableaux of shape $\pi$ ([Ful97, §4.3]):

$$
f^{\pi}=\frac{n !}{\prod_{(i, j) \in \pi} h_{\pi}(i, j)}
$$

where $h_{\pi}(i, j)$ denotes the number of boxes in the hook of $(i, j)$ in $\pi$, i.e. in formulas $h_{\pi}(i, j)=$ $\mid\{(a, b) \in \pi \mid(a=i$ and $b \geqslant j)$ or $(a \geqslant i$ and $b=j)\} \mid$. The following corollary shows that the hook length formula gives the answer to some counting problems related to $p$-cells:

Corollary 4.37. Let $C$ be a two-sided $p$-cell. Denote by $\pi$ the shape of the $P$ and $Q$-symbols of the elements in $C$. Then the following holds:

(i) The number of left (or right) p-cells in $C$ is given by $f^{\pi}$.

(ii) For any left (or right) $p$-cell contained in $C$, the corresponding p-cell module is free of rank $f^{\pi}$ over $\mathbb{Z}\left[v, v^{-1}\right]$.

Observe that the preorder $\underset{L R}{\stackrel{p}{\leqslant}}$ is by definition generated by $\underset{L}{\stackrel{p}{\leqslant}}$ and $\underset{R}{\stackrel{p}{\leqslant}}$. It is in general not clear though whether $\underset{L R}{\stackrel{p}{\sim}}$ is also generated by $\underset{L}{\stackrel{p}{\sim}}$ and $\underset{R}{\stackrel{p}{\sim}}$. For the Kazhdan-Lusztig cell preorder this follows using certain properties of Lusztig's a-function (see [Lus03, Conjectures 14.2 P9 and P10]).

Corollary 4.38. The cell preorder $\underset{L R}{\stackrel{p}{\sim}}$ is generated by $\underset{L}{\stackrel{p}{\sim}}$ and $\underset{R}{\stackrel{p}{\sim}}$ in type $A$.

As a consequence of Lemma 4.21, we get the following result which is known for Kazhdan-Lusztig left cell modules (see [BB05, Theorem 6.5.2]) and for which the proof works exactly as in characteristic 0 :

Corollary 4.39. Let $C_{1}$ and $C_{2}$ be left p-cells in the same two-sided p-cell. Then the corresponding left cell modules are isomorphic.

We want to conclude with some interesting questions that merit further study. We hope to come back to them in another paper:

- In type $A$, the $p$-canonical basis for various primes $p$ gives a family of interesting bases of each Kazhdan-Lusztig cell module. Which bases of the corresponding irreducible representation of $S_{n}$ do they specialize to? In [Wil15, $\S 1$ and $\S 2.3$ ] Williamson explains that any $p$-canonical basis element of a right (or two-sided) cell module that differs from the corresponding KazhdanLusztig basis element provides an example of a reducible characteristic variety of a simple highest weight module for $s l_{n}(\mathbb{C})$.

- In Type $D$, the generalized $\tau$-invariant can be strengthened to give a complete invariant of Kazhdan-Lusztig cells. This is verified in [Gar] together with work of McGovern and Pietraho. Can one show that all elements of a left $p$-cell have the same strengthened generalized $\tau$ invariant to get that Kazhdan-Lusztig cells decompose into $p$-cells in type $D$ ? 


\section{References}

[AHR17] P. N. Achar, W. Hardesty, and S. Riche. On the Humphreys Conjecture on support varieties of tilting modules. 2017. eprint: arXiv:1707.07740.

[AHR18] P. N. Achar, W. Hardesty, and S. Riche. Conjectures on tilting modules and antispherical p-cells. 2018. eprint: arXiv:1812.09960.

[AMRW17a] P. N. Achar, S. Makisumi, S. Riche, and G. Williamson. Free-monodromic mixed tilting sheaves on flag varieties. 2017. eprint: arXiv:1703.05843.

[And04] H. H. Andersen. "Cells in affine Weyl groups and tilting modules". In: Representation theory of algebraic groups and quantum groups. Vol. 40. Adv. Stud. Pure Math. Math. Soc. Japan, Tokyo, 2004, pp. 1-16.

[Ari00] S. Ariki. "Robinson-Schensted correspondence and left cells". In: Combinatorial methods in representation theory (Kyoto, 1998). Vol. 28. Adv. Stud. Pure Math. Kinokuniya, Tokyo, 2000, pp. 1-20.

[BV83] D. Barbasch and D. Vogan. "Primitive ideals and orbital integrals in complex exceptional groups". In: J. Algebra 80.2 (1983), pp. 350-382.

[Bé89] R. Bédard. "Left V-cells for hyperbolic Coxeter groups". In: Comm. Algebra 17.12 (1989), pp. 2971-2997.

[Bel04] M. Belolipetsky. "Cells and representations of right-angled Coxeter groups". In: Selecta Math. (N.S.) 10.3 (2004), pp. 325-339.

[BB05] A. Björner and F. Brenti. Combinatorics of Coxeter groups. Vol. 231. Graduate Texts in Mathematics. Springer, New York, 2005, pp. xiv+363.

[BG15] C. Bonnafé and M. Geck. "Hecke algebras with unequal parameters and Vogan's left cell invariants". In: Representations of reductive groups. Vol. 312. Progr. Math. Birkhäuser/Springer, Cham, 2015, pp. 173-187.

[Dav08] M. W. Davis. The geometry and topology of Coxeter groups. Vol. 32. London Mathematical Society Monographs Series. Princeton University Press, Princeton, NJ, 2008, pp. xvi+584.

[Dye91] M. Dyer. "On the "Bruhat graph" of a Coxeter system". In: Compositio Math. 78.2 (1991), pp. 185-191.

[Eli16] B. Elias. "The two-color Soergel calculus". In: Compos. Math. 152.2 (2016), pp. 327398.

[EK10] B. Elias and M. Khovanov. "Diagrammatics for Soergel categories". In: Int. J. Math. Math. Sci. (2010), Art. ID 978635, 58.

[EW14] B. Elias and G. Williamson. "The Hodge theory of Soergel bimodules". In: Ann. of Math. (2) 180.3 (2014), pp. 1089-1136.

[EW16] B. Elias and G. Williamson. "Soergel calculus". In: Represent. Theory 20 (2016), pp. 295-374.

[Ful97] W. Fulton. Young tableaux. Vol. 35. London Mathematical Society Student Texts. With applications to representation theory and geometry. Cambridge University Press, Cambridge, 1997, pp. $\mathrm{x}+260$.

[Gar] D. Garfinkle. On the classification of primitive ideals for complex classical Lie algebras. $I V$. In preparation.

[Gar90] D. Garfinkle. "On the classification of primitive ideals for complex classical Lie algebras. I". In: Compositio Math. 75.2 (1990), pp. 135-169. 
[Gar92] D. Garfinkle. "On the classification of primitive ideals for complex classical Lie algebra. II". In: Compositio Math. 81.3 (1992), pp. 307-336.

[Gar93] D. Garfinkle. "On the classification of primitive ideals for complex classical Lie algebras. III". In: Compositio Math. 88.2 (1993), pp. 187-234.

[GM88] A. M. Garsia and T. J. McLarnan. "Relations between Young's natural and the Kazhdan-Lusztig representations of $S_{n}$ ". In: Adv. in Math. 69.1 (1988), pp. 32-92.

[Gec03] M. Geck. "On the induction of Kazhdan-Lusztig cells". In: Bull. London Math. Soc. 35.5 (2003), pp. 608-614.

[GH15] M. Geck and A. Halls. "On the Kazhdan-Lusztig cells in type E 8 ". In: Math. Comp. 84.296 (2015), pp. 3029-3049.

[HK83] R. Hotta and N. Kawanaka. Open problems in algebraic groups. Proc. Sympos. on Algebraic Groups and their Representations held at Katata, August 29 - September 3, 1983. Tohoku University, 1983.

[Hum90] J. E. Humphreys. Reflection groups and Coxeter groups. Vol. 29. Cambridge Studies in Advanced Mathematics. Cambridge: Cambridge University Press, 1990, pp. xii+204.

[JW17] L. T. Jensen and G. Williamson. "The p-canonical basis for Hecke algebras". In: Categorification and higher representation theory. Vol. 683. Contemp. Math. Amer. Math. Soc., Providence, RI, 2017, pp. 333-361.

[KL79] D. Kazhdan and G. Lusztig. "Representations of Coxeter groups and Hecke algebras". In: Invent. Math. 53.2 (1979), pp. 165-184.

[KL80] D. Kazhdan and G. Lusztig. "Schubert varieties and Poincaré duality". In: Geometry of the Laplace operator (Proc. Sympos. Pure Math., Univ. Hawaii, Honolulu, Hawaii, 1979). Proc. Sympos. Pure Math., XXXVI. Amer. Math. Soc., Providence, R.I., 1980, pp. 185-203.

[Knu70] D. E. Knuth. "Permutations, matrices, and generalized Young tableaux". In: Pacific J. Math. 34 (1970), pp. 709-727.

[Lus03] G. Lusztig. Hecke algebras with unequal parameters. Vol. 18. CRM Monograph Series. American Mathematical Society, Providence, RI, 2003, pp. vi+136.

[Lus18] G. Lusztig. Comments on my papers. 2018. eprint: arXiv:1707.09368.

[Lus83] G. Lusztig. "Some examples of square integrable representations of semisimple $p$-adic groups". In: Trans. Amer. Math. Soc. 277.2 (1983), pp. 623-653.

[Lus85] G. Lusztig. "Cells in affine Weyl groups". In: Algebraic groups and related topics (Kyoto/Nagoya, 1983). Vol. 6. Adv. Stud. Pure Math. Amsterdam: North-Holland, 1985, pp. 255-287.

[Lus87] G. Lusztig. "Cells in affine Weyl groups. II". In: J. Algebra 109.2 (1987), pp. 536-548.

[LW18] G. Lusztig and G. Williamson. "Billiards and tilting characters for $\mathrm{SL}_{3}$ ". In: SIGMA Symmetry Integrability Geom. Methods Appl. 14 (2018), Paper No. 015, 22.

[Mat94] A. Mathas. "Some generic representations, W-graphs, and duality". In: J. Algebra 170.1 (1994), pp. 322-353.

[MP05] T. P. McDonough and C. A. Pallikaros. "On relations between the classical and the Kazhdan-Lusztig representations of symmetric groups and associated Hecke algebras". In: J. Pure Appl. Algebra 203.1-3 (2005), pp. 133-144.

[McG96] W. M. McGovern. "Left cells and domino tableaux in classical Weyl groups". In: Compositio Math. 101.1 (1996), pp. 77-98. 
[Roi98] Y. Roichman. "Induction and restriction of Kazhdan-Lusztig cells". In: Adv. Math. 134.2 (1998), pp. 384-398.

[Shi86] J.-Y. Shi. The Kazhdan-Lusztig cells in certain affine Weyl groups. Vol. 1179. Lecture Notes in Mathematics. Springer-Verlag, Berlin, 1986, pp. $\mathrm{x}+307$.

[Shi88] J.-Y. Shi. "A two-sided cell in an affine Weyl group. II". In: J. London Math. Soc. (2) 37.2 (1988), pp. 253-264.

[Shi94] J.-Y. Shi. "Left cells in affine Weyl groups". In: Tohoku Math. J. (2) 46.1 (1994), pp. 105-124.

[Shi02] J.-Y. Shi. "Coxeter elements and Kazhdan-Lusztig cells". In: J. Algebra 250.1 (2002), pp. 229-251.

[Shi06] J.-Y. Shi. "Left cells containing a fully commutative element". In: J. Combin. Theory Ser. A 113.3 (2006), pp. 556-565.

[Soe97] W. Soergel. "Kazhdan-Lusztig-Polynome und eine Kombinatorik für Kipp-Moduln". In: Represent. Theory 1 (1997), 37-68 (electronic).

[Tit89] J. Tits. "Groupes associés aux algèbres de Kac-Moody". In: Astérisque 177-178 (1989). Séminaire Bourbaki, Vol. 1988/89, Exp. No. 700, 7-31.

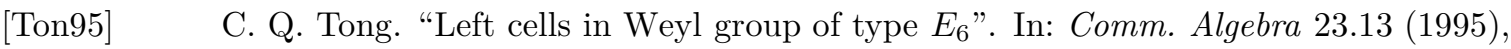
pp. 5031-5047.

[Vog79] D. A. Vogan Jr. "A generalized $\tau$-invariant for the primitive spectrum of a semisimple Lie algebra". In: Math. Ann. 242.3 (1979), pp. 209-224.

[Vog80] D. A. Vogan Jr. "Ordering of the primitive spectrum of a semisimple Lie algebra". In: Math. Ann. 248.3 (1980), pp. 195-203.

[Wil03] G. Williamson. "Why the Kazhdan-Lusztig basis of the Hecke algebra of type $A$ is cellular". Available at http://people.mpim-bonn.mpg.de/geordie/Hecke.pdf. $10 / 2003$.

[Wil15] G. Williamson. "A reducible characteristic variety in type $A$ ". In: Representations of reductive groups. Vol. 312. Progr. Math. Birkhäuser/Springer, Cham, 2015, pp. 517532.

[Wil17a] G. Williamson. "On torsion in the intersection cohomology of Schubert varieties". In: J. Algebra 475 (2017), pp. 207-228.

[Wil17b] G. Williamson. "Schubert calculus and torsion explosion". In: J. Amer. Math. Soc. 30.4 (2017). With a joint appendix with Alex Kontorovich and Peter J. McNamara, pp. 1023-1046.

[Xi89] N. H. Xi. "An approach to the connectedness of the left cells in affine Weyl groups". In: Bull. London Math. Soc. 21.6 (1989), pp. 557-561.

Université Clermont Auvergne, CNRS, LMBP, F-63000 Clermont-Ferrand, France

E-mail address, Lars Thorge Jensen: lars_thorge.jensen@uca.fr 